\title{
Kinetic Theory of Runaway Air-Breakdown
}

\author{
R.A. Roussel-Dupré \\ Space Sciences and Technology Division, Los Alamos National Laboratory, \\ Los Alamos, NM 87545 \\ A.V. Gurevich \\ P.N. Lebedev Institute of Physics, Moscow 117924, Russia \\ T. Tunnell \\ EG\&G Energy Measurements, Inc., Los Alamos, NM 87544
}

RECEIVED SEP 20993

\author{
G.M. Milikh \\ Department of Astronomy, University of Maryland, College Park, MD 29742
}

The kinetic theory for a new air breakdown mechanism advanced in a previous paper is developed. The relevant form of the Boltzmann equation is derived and the particle orbits in both velocity space and configuration space are computed. A numerical solution of the Boltzmann equation, assuming a spatially uniform electric field, is obtained and the temporal evolution of the electron velocity distribution function is described. The results of our analysis are used to estimate the magnitude of potential $\mathbf{x}$-ray emissions from discharges in thunderstonns.

\section{DISCLAIMER}

\begin{abstract}
This report was prepared as an account of work sponsored by an agency of the United States Government. Neither the United States Government nor any agency thereof, nor any of their employees, makes any warranty, express or implied, or assumes any legal liability or responsibility for the accuracy, completeness, or usefulness of any information, apparatus, product, or process disclosed, or represents that its use would not infringe privately owned rights. Reference herein to any specific commercial product, process, or service by trade name, trademark, manufacturer, or otherwise does not necessarily constitute or imply its endorsement, recommendation, or favoring by the United States Government or any agency thereof. The views and opinions of authris expressed herein do not necessarily state or reflect those of the United States Government or any agency thereof.
\end{abstract}




\section{INTRODUCTION}

In a previous paper ${ }^{i}$ (hereafter, Paper $I$ ) we described a new mechanism for air breakdown in which high-energy electrons in the hundreds of $\mathrm{keV}$ range are accelerated by thunderstorm electric fields to relativistic energies (approximating 'runaway'), producing secondary electrons (in the tens, hundreds of $\mathrm{keV}$ range) which in turn runaway in energy and create additional secondaries. This process leads to an avalanche of the high-energy electron population, resulting in the formation of an electron beam, and requires large scale lengths to develop (hundreds of meters) at atmospheric pressure. In the presence of thunderstorm electric fields the beam propagates through the atmosphere over hundreds of meters to kilometer scale lengths and ultimately deposits a significant amount of charge outside of the region where the threshold for initiation of this mechanism is exceeded. While runaway breakdown has obvious implications for lightning initiation, it is clear that this process is a fundamental, new plasma effect that could have its manifestation in many different natural phenomena. Because of the large scale lengths necessary to produce an observable effect, however, laboratory breakdown experiments have not to date identifed the presence of this mechanism. Indeed, it will be necessary to perform experiments at very high atmospheric pressures in order to study this process in the laboratory. In the absence of such experiments, it is instructive to examine runaway breakdown in the context of speculations advanced concerning the production of high-energy secondary electrons in thunderstorms and to mention the recent $x$-ray measurements which clearly suggest that this mechanism is at work in thunderstorms.

The notion that secondary electrons generated by cosmic rays could be accelerated over large distances (kilometers) by thunderstorm electric fields was first proposed by Wilson 2,3 in 1924 . Since that time a number of experiments have been performed to look for the presence of high-energy electrons in the electrical discharges of thunderstorms, either by detecting them directly or by measuring the corresponding bremsstrahlung radiation emitted by these electrons. The early results were conflicting, however, in the last twenty-five years there have been a number of intriguing developments. In 1967 Shaw ${ }^{4}$ reported a general increase in count rates associated with nearby thunderstorm activity as measured by a scintillation counter fielded on Mt. Lemmon, Arizona. In 1979, Whitmire 5 attributed the enhanced radiation measured by dosimeters placed on a 500-m tower to $x$-rays produced by lightning. More recently, Parks et al. 6 (1981) and McCarthy and Parks ${ }^{7}$ (1985) have flown x-ray spectrometers through thunderstorms and observed sharp increases in the $x$-ray flux from 3 to $110 \mathrm{keV}$ prior to lightning strokes. Noting that a substantial population of cosmic ray secondary electrons could be maintained by thunderstorm electric fields, McCarthy and Parks 8 investigated the possibility that bremsstrahlung emission associated with these high-energy electrons could be the source of the enhanced $x$-ray flux. They concluded however that, under the most favorable conditions, this mechanism could not account for the measured level of $x$-rays. A review of this subject was provided by D'Angelo ${ }^{9}$ (1987) who also reported observing a pronounced increase in the number of electron tracks in a diffusion cloud chamber coincident with nearby lightning flashes. 
In this paper the kinetic theory for the air breakdown mechanism advanced in Paper I is developed. The relevant form of the Boltzmann equation is derived in Sec. II while the particle orbits in both velocity space and configuration space are obtained in Sec. III. A numerical solution of the Boltzmann equation, assuming a spatially uniform electric field, is presented in Sec. IV. In Sec. V, the results of our analysis are used to estimate the magnitude of potential $x$-ray emissions. It is shown that the details of electron beam formation and charge deposition could well account for the observed $x$-ray fluxes measured by Shaw and McCarthy and Parks. Concluding remarks are provided in Sec. VI.

\section{BOLTZMANN EQUATION}

The Boltzmann equation for the electron velocity distribution function at high-energies $(\varepsilon>10$ $\mathrm{keV}$ ) with a uniform, externally applied electric field can be written:

$$
\frac{\partial f}{\partial t}-\left[\frac{\left(1-\mu^{2}\right)}{p} \frac{\partial f}{\partial \mu}+\mu \frac{\partial f}{\partial p}\right] e E=\frac{\partial_{e} f}{\partial t}
$$

where $f$ is the distribution function, $p$ is the magnitude of the electron momentum, $\mu$ is the cosine of the angle between the electric field and the momentum vector (see Fig. 1), $E$ is the magnitude of the applied electric field, and $\frac{\partial_{\mathrm{e}} \mathrm{f}}{\partial t}$ is the Boltzmann collision integral for electron-air interactions. At high energies, electron-air interactions are primarily Coulomb in nature and the electrons undergo small deflections in both velocity space and configuration space. In this limit, the collision integral can be written,

$$
\begin{aligned}
\frac{\partial_{\mathrm{e} f}}{\partial \mathrm{t}}= & \frac{1}{\mathrm{p}^{2}} \frac{\partial\left(\mathrm{p}^{2} \mathrm{~F}_{\mathrm{D} f}\right)}{\partial \mathrm{p}}+\frac{\left(\frac{\mathrm{Z}}{2}+1\right) \mathrm{F}_{\mathrm{D}}}{4 \gamma \mathrm{p}} \frac{\partial}{\partial \mu}\left[\left(1-\mu^{2}\right) \frac{\partial \mathrm{f}}{\partial \mu}\right] \\
& +N_{\mathrm{m} \beta c} \int \mathrm{d} \Omega \int_{\varepsilon_{L}}^{\infty} d \varepsilon^{\cdot}\left(\frac{\gamma^{\prime 2}-1}{\gamma^{2}-1}\right) f\left(\varepsilon^{\prime}, \mu^{\circ}\right) \sigma\left(\varepsilon^{\prime}, \varepsilon, \xi\right),
\end{aligned}
$$

where $F_{D}$ is the dynamical friction force first obtained by Bethe ${ }^{10}$; namely, 


$$
\begin{aligned}
& F_{D}=\frac{4 \pi Z e^{4} N_{m}}{m c^{2}} \frac{\gamma^{2}}{\gamma^{2}-1} \\
& \text { - }\left\{\ln \left[\frac{m c^{2}\left(\gamma^{2}-1\right)^{1 / 2}(\gamma-1)^{1 / 2}}{\sqrt{2} I}\right]-\left(\frac{2}{\gamma}-\frac{1}{\gamma^{2}}\right) \frac{\ln 2}{2}+\frac{1}{2 \gamma^{2}}+\frac{(\gamma-1)^{2}}{16 \gamma^{2}}\right\} \text {, }
\end{aligned}
$$

$\mathrm{Z}$ ( $=14.5$ ) is the mean molecular charge for air, $\mathrm{I}=80.5 \mathrm{eV}$ for air, $\mathrm{e}$ is the electron charge, $\mathrm{c}$ is the speed of light, $m$ is the electron mass, $N_{m}$ is the molecular density, $\gamma=\frac{1}{\sqrt{1-\beta^{2}}}, \beta=\frac{v}{c}, v$ is the electron speed, $\varepsilon$ $\left[=(\gamma-1) \mathrm{mc}^{2}\right]$ is the electron kinetic energy, $\sigma\left(\varepsilon^{\prime}, \varepsilon, \xi\right)$ is the doubly differential ionization cross-section for the production of secondary electrons of energy $\varepsilon \leq \frac{\varepsilon^{\prime}-\varepsilon_{t}}{2}, \varepsilon_{\mathrm{l}}(=15 \mathrm{eV})$ is equal to the mean threshold energy for ionization in air, and we are integrating over all incident electrons of energy $\varepsilon^{\prime}$ greater than $\varepsilon_{L}$ and over all solid angles $d \Omega$ into which the incident electron is scattered. A plot of the dynamical friction force (normalized to its minimum value) as a function of energy $\varepsilon$ is provided in Fig. 2. Note that a minimum exists at $\varepsilon_{\min }=1.4 \mathrm{MeV}$ corresponding to $\gamma_{\min }=3.8$.

The doubly differential ionization cross-section is derived from the work of Moller ${ }^{11,12}$ which assumes that the dominant ionization mechanism involves Coulomb interactions between pairs of free electrons. The cross-section is written,

$$
\begin{aligned}
\sigma\left(\varepsilon^{\prime}, \varepsilon, \xi\right)= & \frac{2 \pi \mathrm{Z} \mathrm{e}^{4}}{\mathrm{mc^{2 }}} \frac{\gamma^{\prime 2}}{\gamma^{\prime 2}-1} \\
& \cdot\left[\frac{1}{\varepsilon^{2}}-\frac{1}{\varepsilon\left(\varepsilon^{\prime}-\varepsilon\right)} \frac{\left(2 \varepsilon^{\prime}+m c^{2}\right) m c^{2}}{\left(\varepsilon^{\prime}+m c^{2}\right)^{2}}+\frac{1}{\left(\varepsilon^{\prime}-\varepsilon\right)^{2}}+\frac{1}{\left(\varepsilon^{\prime}+m c^{2}\right)^{2}}\right] \frac{\delta\left(\cos \xi-\mu_{0}\right)}{2 \pi} .
\end{aligned}
$$

with $\varepsilon^{\prime}$ equal to the energy of the incident electron, $\varepsilon$ equal to the energy of the secondary electron, $\xi$ equal to the angle between the incident electron and the scattered primary electron [see Figs. 3(a) and 3(b) for a sketch of the collision geometry], and where $\mu_{0}$ is given by,

$$
\mu_{0}=\sqrt{\frac{\varepsilon\left(\varepsilon^{\prime}+2 m c^{2}\right)}{\varepsilon^{\prime}\left(\varepsilon+2 m c^{2}\right)}},
$$

as required by energy and momentum conservation, assuming that the recoil of the molecule can be neglected (see Bethe and Ashkin ${ }^{11}$ ). Substituting Eq. (4) into Eq. (2) yields, 


$$
\begin{aligned}
\frac{\partial_{\mathrm{e}} f}{\partial t}= & \frac{1}{p^{2}} \frac{\partial\left(p^{2} F_{D} f\right)}{\partial p}+\frac{\left(\frac{Z}{2}+1\right) F_{D}}{4 \gamma p} \frac{\partial}{\partial \mu}\left[\left(1-\mu^{2}\right) \frac{\partial f}{\partial \mu}\right] \\
& +N_{m} \beta c \frac{2 \pi Z e^{4}}{m c^{2}} \int_{\varepsilon_{L}}^{\infty} d \varepsilon^{\prime}\left(\frac{\gamma^{\prime 2}-1}{\gamma^{2}-1}\right) \frac{\gamma^{\prime 2}}{\gamma^{2}-1} Q_{M}\left(\varepsilon, \varepsilon^{\prime}\right) \int \frac{d \alpha}{2 \pi} \int d \mu_{\xi} f\left(\varepsilon^{\prime}, \mu^{\prime}\right) \delta\left(\mu_{\xi}-\mu_{0}\right),
\end{aligned}
$$

where the center of mass solid angle $d \Omega$ was expanded into integrals over the component angular coordinates $\alpha$ and $\mu_{\xi}[=\cos \xi$, see Fig. 3( $(\tilde{)})]$ and where $Q_{M}\left(\varepsilon, \varepsilon^{\prime}\right)$ is defined to be,

$$
\mathrm{Q}_{M}\left(\varepsilon, \varepsilon^{\prime}\right)=\left[\frac{1}{\varepsilon^{2}}-\frac{1}{\varepsilon\left(\varepsilon^{\prime}-\varepsilon\right)} \frac{\left(2 \varepsilon^{\prime}+m c^{2}\right) m c^{2}}{\left(\varepsilon^{\prime}+m c^{2}\right)^{2}}+\frac{1}{\left(\varepsilon^{\prime}-\varepsilon\right)^{2}}+\frac{1}{\left(\varepsilon^{\prime}+m c^{2}\right)^{2}}\right]
$$

Given the coordinate system specified in Fig. 3(b), it is possible to express $\mu^{\prime}$ in terms of the three independent variables $\mu, \mu_{\xi}$, and $\alpha$; namely,

$$
\mu^{\prime}=\frac{\mu \mu_{\xi} \pm\left(1-\mu_{\xi}{ }^{2}\right)^{1 / 2} \sin \alpha \sqrt{\mu_{\xi}^{2}-\mu^{2}+\left(1-\mu_{\xi}{ }^{2}\right) \sin ^{2} \alpha}}{\mu_{\xi}^{2}+\left(1-\mu_{\xi}{ }^{2}\right) \sin ^{2} \alpha} \text {. }
$$

Eqs. (6) and (8) are further simplified by requiring azimuthal symmetry about the applied electric field. In this case we need only consider collisions occurring in a plane [e.g. the y-z plane in the lab frame, see Fig. (3b)]. In the center of mass frame we choose the plane defined by $\alpha=\pi / 2$. Substituting the latter result into Eq. (8) and integrating over $\alpha$ and $\mu_{\xi}$ in Eq. (6) we find,

$$
\begin{aligned}
\frac{\partial_{\mathrm{e}} f}{\partial t}= & \frac{1}{\mathrm{p}^{2}} \frac{\partial\left(\mathrm{p}^{2} \mathrm{~F}_{\mathrm{D} f}\right)}{\partial \mathrm{p}}+\frac{\left(\frac{Z}{2}+1\right) F_{D}}{4 \gamma \mathrm{p}} \frac{\partial}{\partial \mu}\left[\left(1-\mu^{2}\right) \frac{\partial \mathrm{f}}{\partial \mu}\right] \\
& +N_{m} \beta c \frac{2 \pi Z e^{4}}{m c^{2}} \int_{\varepsilon_{L}}^{\infty} \mathrm{d} \varepsilon^{\prime}\left(\frac{\gamma^{\prime 2}-1}{\gamma^{2}-1}\right) \frac{\gamma^{\prime 2}}{\gamma^{\prime 2}-1} Q_{M}\left(\varepsilon, \varepsilon^{\prime}\right) \frac{1}{2}\left[f\left(\varepsilon^{\prime}, \mu_{, p}^{\prime}\right)+f\left(\varepsilon^{\prime}, \mu_{-}^{\prime}\right)\right],
\end{aligned}
$$

where $\mu_{ \pm}^{\prime}=\mu_{0} \mu \pm\left(1-\mu_{0}^{2}\right)^{1 / 2}\left(1-\mu^{2}\right)^{1 / 2}$. If we further assume that $\varepsilon \ll<\varepsilon^{\prime}, \mathrm{mc}^{2}$ then $\mu_{0} \rightarrow 0$ [see Eq. (5)] and $\mu_{ \pm}^{\prime} \rightarrow \pm\left(1-\mu^{2}\right)^{1 / 2}$. The latter approximations were used in our calculations.

In order to better comprehend the origin of the right hand side of Eq. (2), it is necessary to elaborate on the derivation. The general form of the collision integral (not given here) is composed of two 
parts. One is a source term that accounts for those collisions that populate a small volume element in velocity space around $\varepsilon$ and $\mu$, while the second corresponds to a sink term that accounts for collisions that depopulate this region. The net result (sum of source and sink terms) of small deflections caused by elastic, inelastic, and ionizing collisions is contained in the first two terms on the right hand side of Eq. (2) while the production of secondary electrons is contained in the third term. We note that the former two terms are consistent with a Fokker-Planck treatment where energy diffusion in velocity space is neglected but angular diffusion is included. The Fokker-Planck equation itself is derivable from the full Boltzmann collision integral (see e.g. Chapman and Cowling ${ }^{13}$ ) in the limit of small deflections. The first term on the right hand side of Eq. (2) considers energy loss resulting from interactions with the electrons surrounding air atoms while the second term considers angle scattering resulting from electron and nuclear interactions. The detailed form of the scattering term is based on an analysis of Compton electron scattering by Longmire and Longley. ${ }^{14}$ In the case of ionizing collisions, the dynamical friction term incorporates the effect of populating or depopulating the local region of velocity space of interest as a result of energy loss by the primary electron, assuming that it loses only a small fraction of its initial energy and experiences only small deviations from its initial trajectory. On the other hand, the third term on the right hand side of Eq. (2) includes the contribution of ionizing collisions in which the secondary electron populates the region in velocity space of interest. The secondary electron in this context is defined to be the electron with the lower energy after collision and the appropriate ionization cross-section is given by Eq. (4) with the lower limit on the integral given by $\varepsilon_{L}=2 \varepsilon+\varepsilon_{t}$. We note that this third collision term in Eq. (2) is crucial to our analysis as it accounts for the contribution of the 'runaway' electrons to the population of low energy electrons.

Finally, we note that the collision terms given by Eq. (2) are all proportional to the neutral density as expected for electron-air interactions. Dividing Eq. (1) through by the neutral density leads to a parameterization of the solution for the electron distribution function in terms of the atmospheric pressure i.e. time will scale as $\mathrm{Pt}$ and the applied field as $\mathrm{E} / \mathrm{P}$ where $\mathrm{P}$ is the atmospheric pressure. The results presented below were obtained for an altitude of $5 \mathrm{~km}\left(\mathrm{~N}_{\mathrm{m}}=1.32 \times 10^{19} \mathrm{~cm}^{-3}, \mathrm{P}=372\right.$ Torr $)$ for direct comparison with thunderstorm parameters but can be scaled in this way to any altitude (moving to higher pressure will lead to shorter time scales while higher equivalent fields will be necessary to obtain the same distribution functions). This same scaling is used to describe swarm experiments. 


\section{PARTICLE ORBITS}

The orbit equations are obtained by first recasting Eqs. (1) and (9) in the form,

$$
\begin{gathered}
\frac{\partial f}{\partial t}-\left[\frac{\left(1-\mu^{2}\right)}{p} \frac{\partial f}{\partial \mu}+\mu \frac{\partial f}{\partial p}\right] e E-F_{D} \frac{\partial f}{\partial p}=\left(\frac{\partial F_{D}}{\partial p}+\frac{2 F_{D}}{p}\right) f+\frac{\left(\frac{Z}{2}+1\right) F_{D}}{4 \gamma p} \frac{\partial}{\partial \mu}\left[\left(1-\mu^{2}\right) \frac{\partial f}{\partial \mu}\right] \\
+N_{m} \beta c \frac{2 \pi Z e^{4}}{m c^{2}} \int_{\varepsilon_{L}}^{\infty} d \varepsilon^{\prime}\left(\frac{\gamma^{\prime 2}-1}{\gamma^{2}-1}\right) \frac{\gamma^{\prime 2}}{\gamma^{\prime 2}-1} Q_{M}\left(\varepsilon, \varepsilon^{\prime}\right) \frac{1}{2}\left[f\left(\varepsilon^{\prime}, \mu_{+}^{\prime}\right)+f\left(\varepsilon^{\prime}, \mu_{-}^{\prime}\right)\right] .
\end{gathered}
$$

The fourth term on the left-hand side of Eq. (10) corresponds to the effect of the dynamical friction force in slowing down high-energy electrons along their trajectory. Because a particle orbit represents the motion of a single electron through phase space we are not concerned with the production and loss of electrons resulting from collisions, i.e. we ignore the first, second, and fourth terms on the right-hand side of Eq. (10). The third term on the right-hand side is also ignored because it involves scattering which simply allows particles to jump from orbit to orbit. Thus, the orbit equations are derived by setting the total differential of the distribution function equal to the left-hand side of Eq. (10) and matching coefficients of the derivatives with the result,

$$
\frac{d p}{d t}=-e E \mu-F_{D}
$$

and

$$
\frac{d \mu}{d t}=\frac{-e E\left(1-\mu^{2}\right)}{p} .
$$

Taking the ratio of Eq. (11) to Eq. (12) and expressing momentum in terms of $\gamma$ yields the equation,

$$
\frac{\mathrm{d} \gamma^{2}}{\mathrm{~d} \mu}-\frac{2 \mu}{\left(1-\mu^{2}\right)}\left(\gamma^{2}-1\right)-\frac{2 \ln \left[\frac{\mathrm{mc} \sqrt{\left(\gamma^{2}-1\right)(\gamma-1)}}{\sqrt{2} \mathrm{I}}\right]}{a \delta_{0}\left(1-\mu^{2}\right)} \gamma^{2}=0 .
$$


where $a \equiv \frac{\gamma_{\min }^{2}}{\gamma_{\min }^{2}-1} \ln \left[\frac{\mathrm{mc}^{2} \sqrt{\left(\gamma_{\min }^{2}-1\right)\left(\gamma_{\min }-1\right)}}{\sqrt{2} \mathrm{I}}\right] \approx 11$ and $\delta_{0} \equiv \frac{\mathrm{eE}}{\mathrm{F}_{\min }}$ with $\mathrm{F}_{\min }=\frac{4 \pi \mathrm{Ze}^{4} \mathrm{~N}_{\mathrm{m} a}}{\mathrm{mc}^{2}}$. In the nonrelativistic limit Eq. (13) reduces identically to Eq. (12) of Paper I with $\delta_{0}$ and $a$ defined in the same way.

The threshold electric field needed to maintain a population of high energy electrons and initiate the avalanche process is defined to be $E_{t}=\frac{F_{\min }}{e}$. Normalizing out the molecular density (or air pressure, $P$ ) we have $\frac{E_{t}}{P}=2.87 \mathrm{~V} \mathrm{~cm}^{-1}$ Torr $^{-1}$. For comparison, the threshold electric field for air breakdown caused by the acceleration of thermal electrons with initial energy $\approx .03 \mathrm{eV}$ is $\frac{\mathrm{E}_{\mathrm{t}}^{*}}{\mathrm{P}}=30 \mathrm{~V} \mathrm{~cm}^{-1} \mathrm{Torr}^{-1}$, a factor of ten greater.

In the low-energy limit i.e. $\gamma<\gamma_{\min }$, the natural $\log$ term is assumed to be a constant over the energy range of interest and it is then possible to obtain an analytic form for the solution to Eq. (13); namely,

$$
\gamma^{2}-1=\frac{\left(\gamma_{i}^{2}-1\right)\left(1-\mu_{i}^{2}\left[\frac{1+\mu_{i}}{1-\mu_{i}}\right]^{-\eta}+2 \eta \int_{\mu_{i}}^{\mu}\left[\frac{1+\mu^{\prime}}{1-\mu^{\prime}}\right]^{-\eta} d \mu^{\prime}\right.}{\left(1-\mu^{2}\right)\left[\frac{1+\mu}{1-\mu}\right]^{-\eta}}
$$

where $\gamma_{i}$ and $\mu_{\mathrm{i}}$ are the initial $\gamma$ and $\mu$ values, and $\eta=\frac{\ln \left[\frac{m c^{2} \sqrt{\left(\gamma_{i}^{2}-1\right)\left(\gamma_{i}-1\right)}}{\sqrt{2} I}\right]}{a \delta_{0}}$.

In the high-energy limit, it is not possible to assume that the natural log term is a constant. Nevertheless, an analytic form can be found for the solution to Eq. (13) assuming $\gamma \gg>1$. The result can be written, 


$$
\gamma^{2}-1=\frac{1}{b} \exp \left(\frac{1+\mu}{1-\mu}\right)^{\eta_{1}}\left\{\begin{array}{c}
\left(\frac{1-\mu_{i}}{1+\mu_{i}}\right)^{\eta_{1}}\left[\ln \left[b\left(\gamma_{i}^{2}-1\right)\right]+\frac{\mu_{i}}{\eta_{1}}\right]-\frac{1}{\eta_{1}}\left(\frac{1-\mu}{1+\mu}\right)^{\eta_{1}} \mu \\
+\frac{1}{\eta_{1}} \int_{\mu_{i}}^{\mu}\left(\frac{1-\mu^{\prime}}{1+\mu^{\prime}}\right)^{\eta_{1}} \mathrm{~d} \mu^{\prime}
\end{array}\right\}
$$

where $\mathrm{b}=\left(\frac{\mathrm{mc}}{\sqrt{2} \mathrm{I}}\right)^{\frac{4}{3}}$, and $\eta_{1}=\frac{3}{4 a \delta_{0}}$.

The equivalent relativistic separatrix or "runaway line" as defined by Eq. (13) of Paper I for the non-relativistic case is obtained by calculating the values of $\gamma_{1}$ and $\mu_{\mathrm{i}}$ from Eq. (14) for which $\gamma>\gamma_{\min }$ and $\mu=-1$. We find,

$$
\mu_{s}=1-2\left[\frac{\eta}{1-\eta} \frac{1}{\gamma_{s}^{2}-1}\right]^{\frac{1}{1+\eta}}
$$

where $\eta$ was defined previously. The importance of the separatrix is that it separates velocity space into two regimes. Those electrons injected at an angle $\theta_{S}$ to the applied electric field with an energy greater than $\varepsilon_{\mathrm{s}}\left[=\left(\gamma_{\mathrm{s}}-1\right) \mathrm{mc}^{2}\right]$ possess trajectories that take them ultimately to infinite energies while those with energies below $\varepsilon_{s}$ possess trajectories leading to zero energy. A plot of $\frac{\varepsilon_{s}}{\mathrm{mc}^{2}}$ vs $\mu_{s}$ for $\delta_{0}=2$ is provided in Fig. 4. A direct comparison with Fig. 2 of Paper I (which has an ordinate equal to $\frac{2 \varepsilon_{\mathrm{s}}}{\mathrm{mc}^{2}}$ in the nonrelativistic regime) indicates that relativistic effects for $\delta_{0} \leq 2$ are important. In the vicinity of $\mu_{s}=0$, the threshold energy for runaway for $\delta_{0}=2$ is larger by a factor of approximately 1.35 compared to the results obtained in Paper I. Plots of $\frac{\varepsilon_{\mathrm{s}}}{\mathrm{mc}^{2}}$ vs $\dot{\delta}_{0}$ for $\mu_{\mathrm{s}}=0$ and $\mu_{\mathrm{s}}=-1$ are given in Figs. 5(a) and $5(\mathrm{~b})$, respectively. The former plot identifies the minimum energy necessary for electrons injected perpendicular to the electric field to 'runaway' in energy. These electrons in principle play an important role in defining the avalanche rate as is shown in Paper I because they are produced by the primary population of runaway electrons moving as a beam antiparallel to the electric field and because they ultimately accelerate into the direction of the beam and thereby add to the beam population. The detailed kinetic results presented below indicate, however, that transport across the separatrix is important and that it is the minimum runaway energy associated with those electrons that are moving antiparallel to the electric field [see Fig. 5(b)] that, for example, defines the ionization rate. 
To further illustrate certain aspects of 'runaway' and particle collimation in configuration space, we have computed electron trajectories in both velocity space and configuration space. Plots of $\gamma-1$ as a function of $\mu$ for two initial energies corresponding to $\varepsilon_{\mathrm{i}}=0.05$ and $0.5 \mathrm{MeV}\left(\gamma_{\mathrm{i}}=1.10\right.$ and 1.98 , respectively) and five initial $\mu$-values corresponding to $\mu_{\mathrm{i}}=-0.8,-0.4,0.0,0.4$, and 0.8 are shown in Figs. $6(\mathrm{a})-6(\mathrm{~b})$ with $\delta_{0}=2.0$. Time increases along the trajectories in these plots from positive $\mu$-values to negative $\mu$-values and the calculations span a maximum time of approximately $2 \mu \mathrm{s}$. For those particles with initial energy below the separatrix $\left(\varepsilon_{s}=110 \mathrm{keV}\right.$ for $\left.\mu=-1, \delta_{0}=2\right)$ we find that the orbits move toward $\mu=-1$ but that the electrons simply lose energy monotonically. For those electrons with initial energy greater than $\varepsilon_{\mathrm{s}}$ and moving counter to the field, the orbits are such that they accelerate and eventually turn into a direction approaching $\mu=-1$. Those electrons moving along the field decelerate initially, however, if their energy is sufficiently high these electrons eventually turn into a direction counter to the field while at lower energies they simply continue to lose energy and leave the energy regime of interest.

The temporal variation of $\gamma$ and $\mu$ along the particle trajectories can be found simply by integrating Eq. (12). Plots of $\gamma-1$ vs $t$ and $\mu$ vs $t$ for the same initial energies and $\mu$-values noted above are shown in Figs. 7(a)-7(b) and 8(a)-8(b) respectively. For initial energies below the threshold (<110 keV) and all corresponding initial $\mu$-values we see that the particle energies decrease monotonically with time. Above the threshold ( $>110 \mathrm{keV}$ ) some or all of the orbits show increasing energy as a function of time. The plots of $\mu$ vs $t$ are straightforward to interpret.

Assuming that the applied field points along the $z$-direction of a cylindrical coordinate system in configuration space, the electron trajectories can be computed from the results obtained above and the additional equations,

$$
\begin{aligned}
& \frac{d z}{d t}=\sqrt{\frac{\gamma^{2}-1}{\gamma^{2}} \mu c,} \\
& \frac{d p}{d t}=\sqrt{\frac{\left(\gamma^{2}-1\right)\left(1-\mu^{2}\right)}{\gamma^{2}}} c .
\end{aligned}
$$

Plots of particle trajectories for an initial energy corresponding to $\varepsilon_{i}=0.5 \mathrm{MeV}$ and five initial $\mu$-values corresponding to $\mu_{\mathrm{i}}=-0.8,-0.4,0.0,0.4$, and 0.8 are shown in Fig. 9 with $\delta_{0}=2.0$. We find that for this initial energy and $\mu \leq 0.0$, the particles turn rapidly into the direction of the electric field, i.e. within 9 m or 30 ns [see e.g., Fig. 8(b)]. 


\section{SOLUTION OF BOLTZMANN EQUATION}

\section{A. Numerical technique}

The temporal evolution of the electron distribution is obtained by solving Eq. (10) numierically on a two dimensional mesh of $\ln (\varepsilon)$ and $\mu$. Our numerical treatment evolves around a Taylor series expansion of the distribution function in time written as,

$$
f(t+\Delta t, \gamma, \mu)=f(t, \gamma, \mu)+\frac{\partial f}{\partial t} \Delta t+\frac{\partial^{2} f}{\partial t^{2}} \frac{\Delta t^{2}}{2}+\cdots .
$$

The Boltzmann equation defines a time derivative operator $L$ which allows us to rewrite Eq. (19) as

$$
f(t+\Delta t, \gamma, \mu)=f(t, \gamma, \mu)+L(f) \Delta t+L[L(f)] \frac{\Delta t^{2}}{2}+\cdots .
$$

where

$$
\begin{aligned}
& L(f) \equiv {\left[\frac{\left(1-\mu^{2}\right)}{\sqrt{\gamma^{2}-1}} \frac{\partial \mathrm{f}}{\partial \mu}+\mu \frac{\sqrt{\gamma^{2}-1}}{\gamma} \frac{\mathrm{mc}}{\varepsilon} \frac{\partial \mathrm{f}}{\partial \ln (\varepsilon)}\right] \frac{\mathrm{eE}}{\mathrm{mc}}+\frac{\sqrt{\gamma^{2}-1}}{\gamma} \frac{\mathrm{FD}_{\mathrm{D}}}{\mathrm{mc}} \frac{\mathrm{mc}}{\varepsilon} \frac{\partial \mathrm{f}}{\partial \ln (\varepsilon)} } \\
&+\left(\frac{\sqrt{\gamma^{2}-1}}{\gamma \mathrm{mc}} \frac{\mathrm{mc}}{\varepsilon} \frac{\partial \mathrm{F}_{\mathrm{D}}}{\partial \ln (\varepsilon)}+\frac{2 \mathrm{~F}_{\mathrm{D}}}{\mathrm{mc} \sqrt{\gamma^{2}-1}}\right) \mathrm{f}+\frac{\left(\frac{\mathrm{Z}}{2}+1\right) \mathrm{F}_{\mathrm{D}}}{4 \mathrm{mc} \sqrt{\gamma^{2}-1}} \frac{\partial}{\partial \mu}\left[\left(1-\mu^{2}\right) \frac{\partial \mathrm{f}}{\partial \mu}\right] \\
&+\mathrm{N}_{\mathrm{m}} \beta \mathrm{c} \frac{2 \pi \mathrm{Z} \mathrm{e}^{4}}{\mathrm{mc^{2 }}} \int_{\ln \left(\varepsilon_{\mathrm{L}}\right)}^{\infty} \mathrm{d} \ln \left(\varepsilon^{\prime}\right) \varepsilon^{\prime}\left(\frac{\gamma^{\prime 2}-1}{\gamma^{2}-1}\right) \frac{\gamma^{\prime 2}}{\gamma^{2}-1} \mathrm{Q}_{\mathrm{M}}\left(\varepsilon, \varepsilon^{\prime}\right) \\
& \bullet \frac{1}{2}\left[\mathrm{f}\left(\varepsilon^{\prime},-\left(1-\mu^{2}\right)^{1 / 2}\right)+\mathrm{f}\left(\varepsilon^{\prime},\left(1-\mu^{2}\right)^{1 / 2}\right)\right],
\end{aligned}
$$

and where we have made use of the fact that $L$ is independent of time. A fourth order finite difference approximation is used to evaluate the $\varepsilon$ - and $\mu$-derivatives while a trapezoidal approximation is used for the integral. Although the Taylor series expansion can be easily taken to any order, our calculations are generally carried to order two. Given an appropriate velocity-space mesh that is capable of resolving the dynamical friction force and the ionization cross-section, the time step $\Delta t$ is then chosen in such a way as 
to insure that the Courant condition is satisfied over the entire grid. We note that this numerical technique for solving the Boltzmann equation has been used previously in the modeling of swarm experiments.

The energy of the lower boundary $\left[\varepsilon_{\mathrm{LB}}=\left(\gamma_{\mathrm{LB}}-1\right) \mathrm{mc}^{2}\right]$ is chosen to be greater than or equal to 10 $\mathrm{keV}$, so that the approximations inherent to the derivation of the dynamical friction force are valid, and less than the lower energy at which the dynamical friction force equals the force exerted by the applied field. The value at the upper boundary $\left[\varepsilon_{U B}=\left(\gamma_{\cup B}-1\right) \mathrm{mc}^{2}\right]$ is chosen large enough for the value of the distribution function to fall off to zero. The distribution function at the lower energy boundary is found by solving the Boltzmann equation in the same way as outlined above except that the $\varepsilon$-derivative is set equal to the derivative calculated at the midpoint between the boundary point and the next energy value. A similar technique is used to calculate the distribution function at $\mu=1$ and -1 , for all $\varepsilon$. These boundary conditions permit particles to flow by deceleration (acceleration) below (above) the lower (upper) energy boundary and for particles to be produced at the lower boundary by ionization.

\section{B. Results}

Results were obtained for three cases corresponding to three values of the parameter $\delta_{0}\left(\equiv E / E_{t}\right)=$ 2,5 , and 8 . The relevant computational parameters used in our calculations for all cases are listed in Table I. The initial distribution function was taken in each case to be isotropic and a Gaussian in energy with a mean energy of $\varepsilon_{\mathrm{m}}=2 \mathrm{MeV}$ and a full width at half-maximum (FWHM) $\varepsilon_{w}=277 \mathrm{keV}$. Three dimensional plots of the distribution function as a function of $\log \varepsilon$ and $\mu$ are shown for six different times in Figs. 10(a)-10( $f$, for $\delta_{0}=2$. After an initial transient phase (first three plots) the distribution function settles into a self-similar solution (last three plots) where the shape remains approximately constant while the number density continues to grow. The shape of the distribution function at late times is determined effectively by the competition between the electric field force and the dynamical friction and by the production of secondary electrons. Above the threshold energy $\left[\varepsilon_{\mathrm{s}}(-1,2)=110 \mathrm{keV}\right]$ electrons accelerate and avalanche. Just below the threshold electrons are decelerating out of that energy range faster than they are produced by ionization and there is a decrease in the electron population. At still lower energies the secondary production rate dominates and the distribution function increases in magnitude. The peak in the distribution function at a $90^{\circ}$ angle to the electric field at low energies $\left[\varepsilon<\varepsilon_{\mathrm{s}}(-1,2)\right]$ is caused by the fact that the avalanching electrons are confined to move along the electric field and are ejecting secondary electrons at $90^{\circ}$ to their direction of motion.

A unique population of electrons which we refer to as the 'beam' is carved out of the distribution function by requiring that their energies lie above the instantaneous threshold defined by

$$
-\mathrm{eE} \mu \geq \mathrm{F}_{\mathrm{D}} \text {. }
$$


Electrons with energy above this threshold are accelerating and turning into the direction antiparallel to the applied electric field. Scattering has the effect of smoothing out or diffusing the sharp boundaries defined by this condition. Plots of the beam distribution obtained in this way are provided in Figs. 11 (a)-11(f) for the same times as presented in Figs. 10(a)-10(f). A more quantitative picture is attained by plotting the beam distribution function vs energy for several values of $\mu$ [see Figs. 12(a)-12(f)]. Note that the bump on the tail of the distribution represents the remnants of the initial Maxwellian distribution function which possessed an initial mean energy of $2 \mathrm{MeV}$.

Plots of the electron density, mean kinetic energy, and energy spread obtained by taking appropriate moments of the distribution function as a function of time are given in Figs. 13(a)-13(c) for the overall distribution function and in Figs. 13(d)-(f) for the beam. The energy spread is defined to be the standard deviation of the distribution function times a factor equal to $\sqrt{\ln 2}$. For a Gaussian distribution the energy spread would be equivalent to the FWHM. From Figs. 13(a) and 13(d) we see that the electron density for both the overall discribution and the beam avalanches after an initial transient phase with an average e-folding time of $27 \mathrm{~ns}$. The mean kinetic eriergy for the overall distribution [Fig. 13(b)] decays from the initial $2 \mathrm{MeV}$ value to a nearly steady-state value of approximately $0.61 \mathrm{MeV}$. Similarly, the mean energy of the beam reaches a nearly constant value of $0.95 \mathrm{MeV}$. The energy spread for both distributions [Figs. 13(c) and 13(f)] increases from the initial value of $277 \mathrm{keV}$ to maximum values of $1.75 \mathrm{MeV}$ (overall) and $2.05 \mathrm{MeV}$ (beam) and subsequently decreases, as the population of low energy secondaries continues to rise, reaching steady-state values of $1.18 \mathrm{MeV}$ (overall) and $1.45 \mathrm{MeV}$ (beam). We note that the energy moments reported here for the overall distribution function are dependent on the energy chosen for the lower boundary. If the range of our calculations were extended to lower energies, we would obtain lower mean energies for the overall distribution function. A summary of the steady-state moments discussed above is provided in Table II.

Similar results were obtained for $\delta_{0}=5$ and 8 . Plots for these cases are not provided, however, a summary of the steady-state results is presented in Table II. The behavior of the distribution function with increasing field strength is tied strongly to the corresponding decrease in threshold energy for beam formation. The latter values for $\delta_{0}=5$ and 8 are $\varepsilon_{s}\left(-1, \delta_{0}\right)=30.0$ and $16.2 \mathrm{keV}$, respectively. As the threshold ener $; y$ decreases the beam electrons occupy a larger regime in phase space (both in angle and energy) and the ionization rate increases because the secondary production rate varies as $1 / \varepsilon^{2}$ [cf. Eq. (2)]. For the range of field strengths discussed in this paper $t_{i}$ is proportionai to $\varepsilon_{s}^{x}\left(-1, \delta_{0}\right)$, where $x$ has a mean value of approximately 1.2 . In the non-relativistic regime $\left(\varepsilon_{\mathrm{s}} \ll \mathrm{mc}^{2}\right.$, or $\left.\delta_{0}>2\right)$, $x$ equals 1.43 . For values of $\delta$ below 1.5 the ionization rate is controlled primarily by the acceleration of electrons into and out of the phase space occupied by the electron beam and as a result the ionization rate decreases substantially relative to that predicted by the latter proportionality. When $\delta_{0}$ is increased from 2 to 5 we see an increase in both the mean energy and energy spread of the beam distribution function. This effect results not only from the fact that at higher fields the beam electrons occupy a larger energy range but also because the electrons can accelerate to higher energies on the same time scale as production of the low 
energy population proceeds. At higher field strengths $\left(\delta_{0}=8\right)$ the production rate of low energy electrons proceeds at a faster rate than does acceleration to higher energy and the mean energy and energy spread of the distribution function decreases. The details of these effects can be seen in the plots of the self-similar beam distribution functions shown for the three values of $\delta_{0}$ in Figs. 14(a)-(c).

\section{X-RAY EMISSIONS}

The doubly-differential cross-section for bremsstrahlung emission by a relativistic electron moving through air was initially derived by Bethe and Heitler 15 and in the limit of complete screening is given by, 16,17

$$
\begin{gathered}
\frac{d^{2} \chi_{R}}{d v d \Omega^{\prime}}=\frac{\frac{Z}{2}\left(\frac{Z}{2}+1\right)}{137 v}\left(\frac{e^{2}}{m^{2}}\right)^{2} 4\left\{\left[1+\left(\frac{\varepsilon}{\varepsilon_{0}}\right)^{2}-\frac{2 \varepsilon}{3 \varepsilon_{0}}\right] \ln \left(183 \mathrm{Z}^{-1 / 3}\right)+\frac{\varepsilon}{9 \varepsilon_{0}}\right\} \\
\cdot \frac{3}{16 \pi}\left[\frac{1}{\gamma^{2}(1-\beta \cos \psi)^{2}}\right]\left[1+\frac{(\cos \psi-\beta)^{2}}{(1-\beta \cos \psi)^{2}}\right],
\end{gathered}
$$

where $v$ is the frequency of the emitted radiation, $\varepsilon_{0}$ is the total energy of the incident electron, $\beta$ and $\gamma$ are determined from $\varepsilon_{0}, \varepsilon=\varepsilon_{0}-h v$, and $\psi$ is the angle between the incident particle momentum and the emitted photon. The specific emissivity (number of photons radiated per unit volume per unit frequency per steradian per second) is then obtained by integrating over the electron distribution function; namely,

$$
\varepsilon_{v}=2 N_{m} \int d p p^{2} \int d \Omega f(p, \mu) \frac{d^{2} \chi_{R}}{d v d \Omega^{\prime}} \beta c .
$$

With these results, the specific intensity at a distance $R$ from the source and an angle $\theta$ ' relative to the applied electric field, can then be approximated as,

$$
I_{v}\left(R, \theta^{\prime}\right)=\frac{\varepsilon_{v} V_{s}}{R^{2}},
$$

where $V_{\mathrm{S}}$ is the volume of the source region. A three-dimensional plot of the specific intensity calculated for $\delta_{0}=2$ and normalized to a distance of $1 \mathrm{~km}$ as a function of the photon energy and emission angle measured relative to the electric field direction is shown in Fig. 15(a). Two-dimensional plots of the intensity vs angle for several photon energies are provided in Fig. 15(b). The relativistic effects that cause forward peaking of the radiation field are readily discemible at high photon energies (e.g. $1 \mathrm{MeV}$ ) where the electrons responsible for this emission have high energies $(>1 \mathrm{MeV})$ and are confined to a narrow 
angular range around the direction antiparallel to the electric field. At low energies the angular dependence becomes less forward peaked in part because the electron distribution function is broader in angle with a maximum at 900 to the electric field.

The results presented in Figs. 15(a) and 15(b) were determined for a time equal to $150 \mathrm{~ns}$ after initiation of the kinetic calculations. For comparison with measurements of $x$-rays in thunderstorms we extrapolate these results to a time $\tau_{b}(\approx 1.2 \mu \mathrm{s})$ correspondiag to a mean risetime and duration for strong breakdown events associated with thunderstorm discharges as observed for example from broad-band radio frequency measurements 18,19 . Assuming that the distribution function is self-similar and exponentiating in magnitude with an ionization time of $27 \mathrm{~ns}$ we obtain a multiplication factor equal to 7.75 $x 10^{16}$ for extrapolation of the results in Fig. (15). The absorption scale length for $100 \mathrm{keV}$ photons in sea-level air is approximately $150 \mathrm{~m}^{20}$ so that the peak intensity observed at $1 \mathrm{~km}$ from the breakdown region is approximately $I_{100 \mathrm{keV}}$ (at $\left.1 \mathrm{~km}\right) \approx 5.9 \times 10^{5}$ photons $/ \mathrm{cm}^{2} / \mathrm{sec} / \mathrm{ster} / \mathrm{eV}$. Assuming a geometrical factor of $40 \mathrm{~cm}^{2}$ - ster, the intensity of $\mathrm{x}$-rays in a $16 \mathrm{keV}$ energy bin around $100 \mathrm{keV}$ at $1 \mathrm{~km}$ from our electron beam source with $\delta_{0}=2$ would be approximately $I_{100 \mathrm{keV}}$ (at $\left.1 \mathrm{~km}\right)=3.1 \times 10^{4}$ photons $/$ event and at $2 \mathrm{~km}$ from the source $I_{100 \mathrm{keV}}($ at $2 \mathrm{~km}) \approx 10$ photons / event. For photon counting systems with sufficient time resolution $(<1 \mu \mathrm{s}$ ), a single strong event of this type occurring within $1 \mathrm{~km}$ of the detector would more than account for the enhanced count rates observed in thunderstorms. ${ }^{6-8}$ It is also possible, however, for 1000 much weaker events occurring within $1 \mathrm{~km}$ of the detector per second and driven by thunderstorm fields such that $\delta_{0}=1.5$ over a $1 \mathrm{~km}$ diameter region with a range of $300 \mathrm{~m}$ to account for the observed count rates. These results of course are strongly dependent on the duration of the discharge and on the attenuation length (inversely proportional to atmospheric pressure) of the $x$-ray photons. The only remaining issue in this regard is a more precise identification of the $x$-ray source. Simultaneous triggered and gated, high-time resolution $(<15 \mu \mathrm{s})$ measurements of $x$-ray, optical, and rf emissions would provide the necessary data for association of the $x$-ray source with a specific lightning mechanism.

\section{CONCLUSIONS}

The single-particle kinetic theory developed in Paper I was based on orbit analysis and a simplified one-particle expression for secondary electron production. A more detailed analysis based on the exact kinetic theory was needed. The kinetic results presented in this paper have yielded some new insights into the details of the runaway breakdown mechanism. First, it is clear that the kinetic effects associated with electron transport in velocity space around the separatrix play an essential role in defining the avalanche rate. In particular, the critical energy which marks the transition to the runaway regime and defines the avalanche rate is found to be $\varepsilon_{s}\left(-1, \delta_{0}\right)$. In addition we find that angle scattering across the separatrix can also be important. Second, the detailed shape of the distribution function yields a more quantitative picture for the extent of the runaway electron beam in both energy and angle and provides a basis for estimating the spatial dimensions of the electron beam as a function of time. The latter information was used to estimate the $x$-ray flux expected from thunderstorm discharges. The results indicate that runaway 
breakdown can easily account for the $\mathrm{x}$-ray observations of McCarthy and Parks 7,8 . Future publications will address the relevance of the runaway mechanism to lightning initiation.

Finally, we hope that the theoretical results presented in this paper and Paper I will provide new incentive for further examination of lightning discharges as a whole occurring both on earth and in other planetary systems and that corroborating laboratory measurements of this new breakdown mechanism will be performed in the near future.

\section{ACKNOWLEDGMENTS}

The authors would like to thank Dr. C. Rhodes for helpful discussions regarding lightning phenomenology and Dr. G. Shaw for providing a historical perspective on the measurements of runaway electrons. This work was performed under the auspices of the US Department of Energy and supported in part by Office of Naval Research (ONR) contract \# N0001490K2010. 


\section{References}

${ }^{1}$ A.V. Gurevich, G.M. Milikh, R.A. Roussel-Dupré, Phys. Lett. A 165, 463 (1992).

${ }^{2}$ C.T.R. Wilson, Proc. Cambridge Phil. Soc. 22, 534 (1924).

${ }^{3}$ C.T.R. Wilson, Proc. Phys. Soc. (London) 37, 320 (1925).

${ }^{4}$ G.E. Shaw, J. Geophys. Res. 72, No. 18, 4623 (1967).

5D.P. Whitmire, Lett. Nuovo Cimiento 26, 497 (1979).

6G.K. Parks, B.H. Mauk, R. Spiger, and J. Chin, Geophys. Res. Lett. 8, No. 11, 1176 (1981).

${ }^{7}$ M.P. McCarthy and G.K. Parks, Geophys. Res. Lett. 12, No. 6, 393 (1985).

8M.P. McCarthy and G.K. Parks, J. Geophys. Res. 97, No. D5, 5857 (1992).

${ }^{9}$ N. D'Angelo, Ann. Geophysicae 5B, (2), 119 (1987).

${ }^{10}$ H. A. Bethe, Ann. Phys. 5, 325 (1930).

${ }^{11}$ H.A. Bethe and J. Ashkin, in Experimental Nuclear Physics, edited by E. Segrè, 1, (John Wiley and Sons, New York, 1953), p. 277.

12C. Molier, Ann. Physik, 14, 531 (1932).

${ }^{13}$ S. Chapman and T.G. Cowling, The Mathematical Theory of Non-Uniform Gases, (Cambridge University Press, London, 1970), p. 400.

${ }^{14}$ C.L. Longmire and H.J. Longley, Defense Nuclear Agency report no. DNA 3192T (1973).

${ }^{15}$ H.A. Bethe and W. Heitler, Proc. Roy. Soc. (London) A 146, 83 (1934).

16H.A. Bethe and J. Ashkin, in Experimental Nuclear Physics, edited by E. Segrè, 1, (John Wiley and Sons, New York, 1953), p. 260.

17J.D. Jackson, Classical Electrodynamics, Second Edition (John Wiley and Sons, New York, 1975), p. 713.

18B.P. Kuhlman. M.J. Reazer, and P.L. Rustan, 1981 WC-130 Airborne Lightning Characterization Program Data Review, Air Force Wright Aeronautical Laboratories Report, AFWAL-TR-84-3024, (1984).

19J.P. Moreau and P.L. Rustan, Electromagnetics 7, 333 (1987).

20In American Institute of Physics Handbook, edited by D.E. Gray, third edition (McGraw Hill, New York, 1972), p. 8-203. 
TABLE I. Computational parameters.

\begin{tabular}{|c|c|c|c|}
\hline Case & $\delta_{0}=2.0$ & $\delta_{0}=5.0$ & $\delta_{0}=8.0$ \\
\hline Altitude $(\mathrm{km})$ & 5.0 & 5.0 & 5.0 \\
$t_{\max }(\mathrm{ns})$ & 150 & 75.0 & 37.5 \\
$\Delta \mathrm{t}(\mathrm{ns})$ & 0.00005 & 0.00005 & 0.00002 \\
$\varepsilon_{\min }(\mathrm{MeV})$ & 0.01 & 0.01 & 0.005 \\
$\varepsilon_{\max }(\mathrm{MeV})$ & 50 & 50 & 100 \\
$\Delta \ln (\varepsilon)$ & 0.08517 & 0.08517 & 0.09903 \\
$\Delta \mu$ & 0.033 & 0.033 & 0.033 \\
\hline
\end{tabular}

TABLE II. Steady-state moments of overall and beam distribution functions.

\begin{tabular}{|c|c|c|c|}
\hline Case & $\delta_{0}=2.0$ & $\delta_{0}=5.0$ & $\delta_{0}=8.0$ \\
\hline & Overall & \multicolumn{2}{|c|}{ Distribution } \\
\hline$t_{i}(n s)$ & 27 & 7.0 & 2.9 \\
\hline$\varepsilon_{\mathrm{m}}(\mathrm{MeV})$ & 0.61 & 1.0 & 0.68 \\
\hline$\varepsilon_{\mathrm{w}}(\mathrm{MeV})$ & 1.18 & 1.8 & 1.3 \\
\hline \multicolumn{4}{|c|}{ Distribution } \\
\hline$t_{i}(n s)$ & 27 & 7.0 & 2.9 \\
\hline$\varepsilon_{m}(\mathrm{MeV})$ & 0.95 & 1.2 & 0.82 \\
\hline$\varepsilon_{\mathrm{w}}(\mathrm{MeV})$ & 1.45 & 1.9 & 1.4 \\
\hline
\end{tabular}




\section{Figure Captions}

FIG. 1. Configuration space geometry. The applied electric field is assumed to be in the z-direction. An electron with momentum $\underline{P}$ is directed at an angle $\theta$ to the field.

FIG. 2. Scaled dynamical friction. The magnitude of the dynamical friction force is plotted as a function of electron energy.

FIG. 3. Ionizing collision geometry. In Figure 3(a) an incident electron of energy $\varepsilon$ ' collides with an air molecule and scatters with energy $\varepsilon_{\mathrm{s}}$. A secondary electron of energy $\varepsilon$ is produced at an angle $\xi$ relative to the incident electron. The azimuthal angle $\alpha$ is measured in the plane perpendicular to that containing the incident and scattered electrons. Conservation of energy requires that $\varepsilon_{s}=\varepsilon^{\prime}-\varepsilon_{\mathrm{t}}-\varepsilon$ where $\varepsilon_{\mathrm{t}}$ is the ionization energy. The equivalent collision geometry in the lab frame is depicted in Figure 3(b). The Zaxis corresponds to the direction of the applied electric field.

FIG. 4. Relativistic Separatrix for $\delta_{0}=2$. The minimum initial electron energy needed for 'runaway' for $\delta_{0}=2$ is plotted as a function of the initial electron direction of motion relative to the applied electric field The parameter $\mu_{\mathrm{s}}$ is defined to be the cosine of the angle between the initial momentum of the electron and the elecuric field.

FIG 5. Minimum energy for 'runaway'. The minimum energy necessary for an electron whose initial momentum is perpendicular [Fig. 5(a)] or antiparallel [Fig. 5(b)] to the electric field to 'runaway' in energy is plotted as a function of $\delta_{0}$.

FIG.6. Particle trajectory in velocity space. The particle kinetic energy normalized to $\mathrm{mc}^{2}$ is plotted as a function of $\mu$ ( = cosine of the angle between the particle momentum and the electric field) for an initial electron kinetic energy of $50 \mathrm{keV}$ [Fig. 6(a)] and $500 \mathrm{keV}$ [Fig. 6(b)] and for five initial $\mu$-values. The electric field strength was taken to be $\delta_{0}=2$.

FIG. 7. Particle orbits in velocity space vs. time. The particle kinetic energy normalized to $\mathrm{mc}^{2}$ is plotted as a function of time (in ns) for an initial electron kinetic energy of $50 \mathrm{keV}$ [Fig. 7(a)] and $500 \mathrm{keV}$ [Fig. 7 (b)] and for five initial $\mu$-values. The electric field strength was taken to be $\delta_{0}=2$.

FIG. 8. Particle orbits in velocity space vs. time. The cosine of the angle between the particle momentum and the electric field is plotted as a function of time (in ns) for an initial electron kinetic energy of $50 \mathrm{keV}$ 
[Fig. 8(a)] and $500 \mathrm{keV}$ [Fig. 8(b)] and for five initial $\mu$-values. The electric field strength was taken to be $\delta_{0}=2$.

FIG. 9. Particle trajectory in configuration space. The electron trajectory in the presence of an electric field applied along the minus z-direction is plotted. Note that the scale in the direction perpendicular to the applied field is much smaller than that along the field. This has the effect of distorting the particle orbit so that the angle between the particle motion and the electric field looks much larger than in reality. The electric field strength was taken to be $\delta_{0}=2$.

FIG. 10. Time history of the overall electron distribution function. The amplitude of the overall electron distribution function obtained for $\delta_{0}=2$ is plotted as a function of $\log \varepsilon$ and $\mu$ for various times including (a) $\mathrm{t}=0.0$, (b) $\mathrm{t}=1.0$, (c) $\mathrm{t}=1.5$, (d) $\mathrm{t}=20$, (e) $\mathrm{t}=50$, and ( $\mathrm{f}$ ) $\mathrm{t}=150 \mathrm{~ns}$.

FIG. 11. Time history of the beam distribution function. The amplitude of the beam distribution function obtained for $\delta_{0}=2$ is plotted as a function of $\log \varepsilon$ and $\mu$ for various times including (a) $t=0.0$, (b) $t=$ 1.0 , (c) $\mathrm{t}=1.5$, (d) $\mathrm{t}=20$, (e) $\mathrm{t}=50$, and $(\mathrm{f}) \mathrm{t}=150$ ns.

FIG. 12. Time history of the beam distribution function. The amplitude of the beam distribution function obtained for $\delta_{0}=2$ is plotted as a function of $\log \varepsilon$ for five angles measured relative to the electric field and for various times including (a) $t=0.0$, (b) $t=1.0$, (c) $t=1.5$, (d) $t=20$, (e) $t=50$, and (f) $t=150$ ns.

FIG. 13. Moments of the overall and beam distribution functions. The overall electron density, mean kinetic energy, and kinetic energy spread are plotted as a function of time in (a)-(c), respectively. The beam electron density, mean kinetic energy, and kinetic energy spread are plotted as a function of time in (d)-(f), respectively.

FIG. 14. Self-similar beam distribution function. The self-similar beam distribution function is plotted as a function of electron energy for five angles measured relative to the applied electric field and for three values of the field including, (a) $\delta_{0}=2$, (b) $\delta_{0}=5$, and (c) $\delta_{0}=8$.

FIG. 15. Bremsstrahlung emission. The specific intensity calculated for $\delta_{0}=2$ and normalized to a distance of $1 \mathrm{~km}$ is plotted in (a) as a function of the log of the photon energy and the emission angle measured relative to the applied field. The specific intensity calculated for $\delta_{0}=2$ and normalized to a distance of $1 \mathrm{~km}$ is plotted in (b) as a function of the emission angle for five photon energies. Atmospheric attenuation is not included in these calculations. 


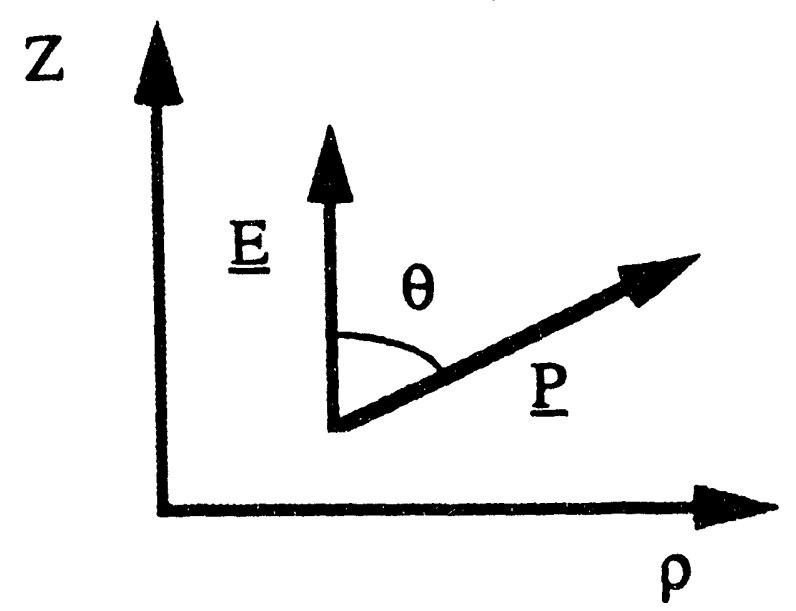

Figure 1. Configuration space geometry. 


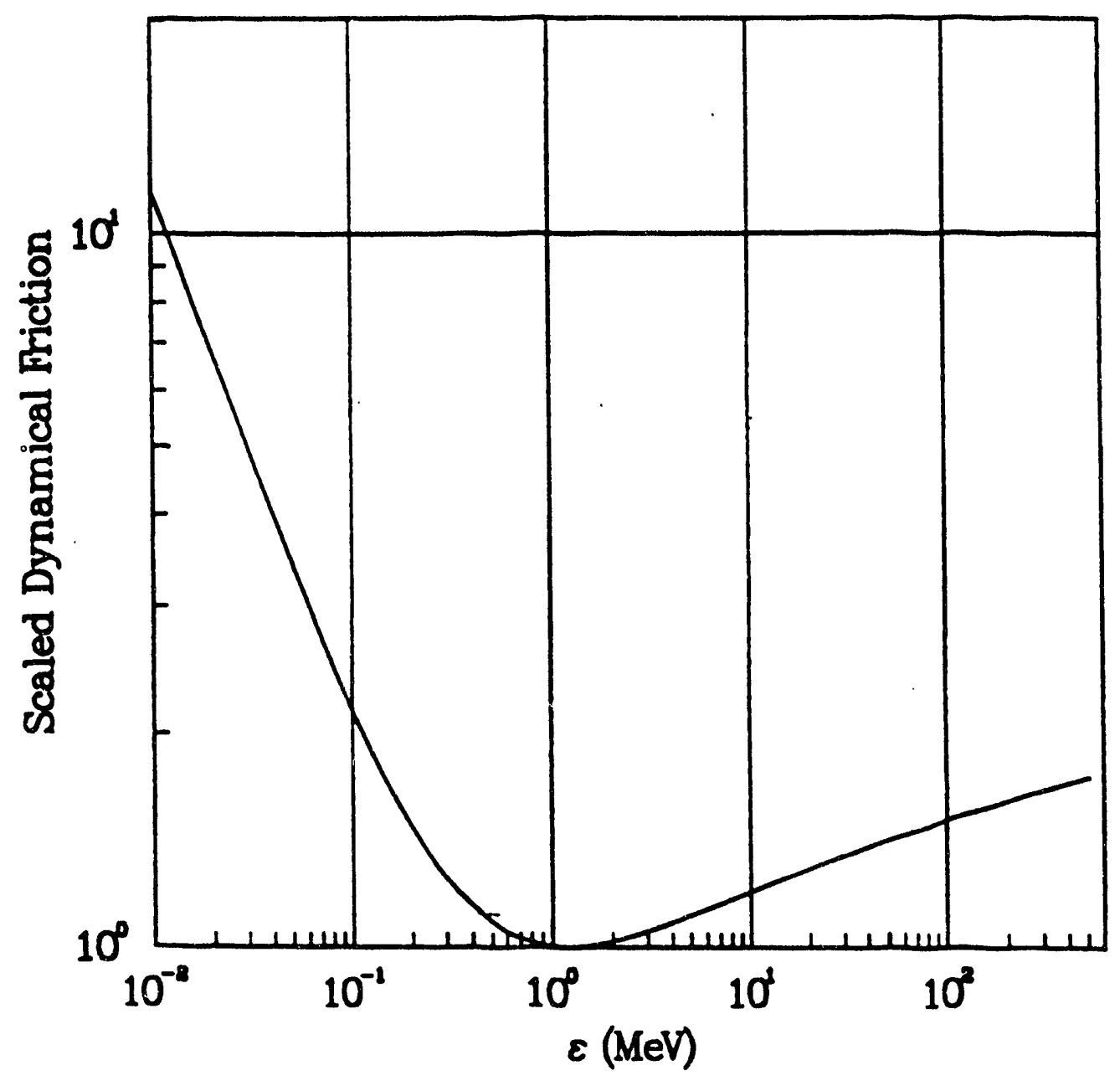

Figure 2. Scaled dynamical friction. 

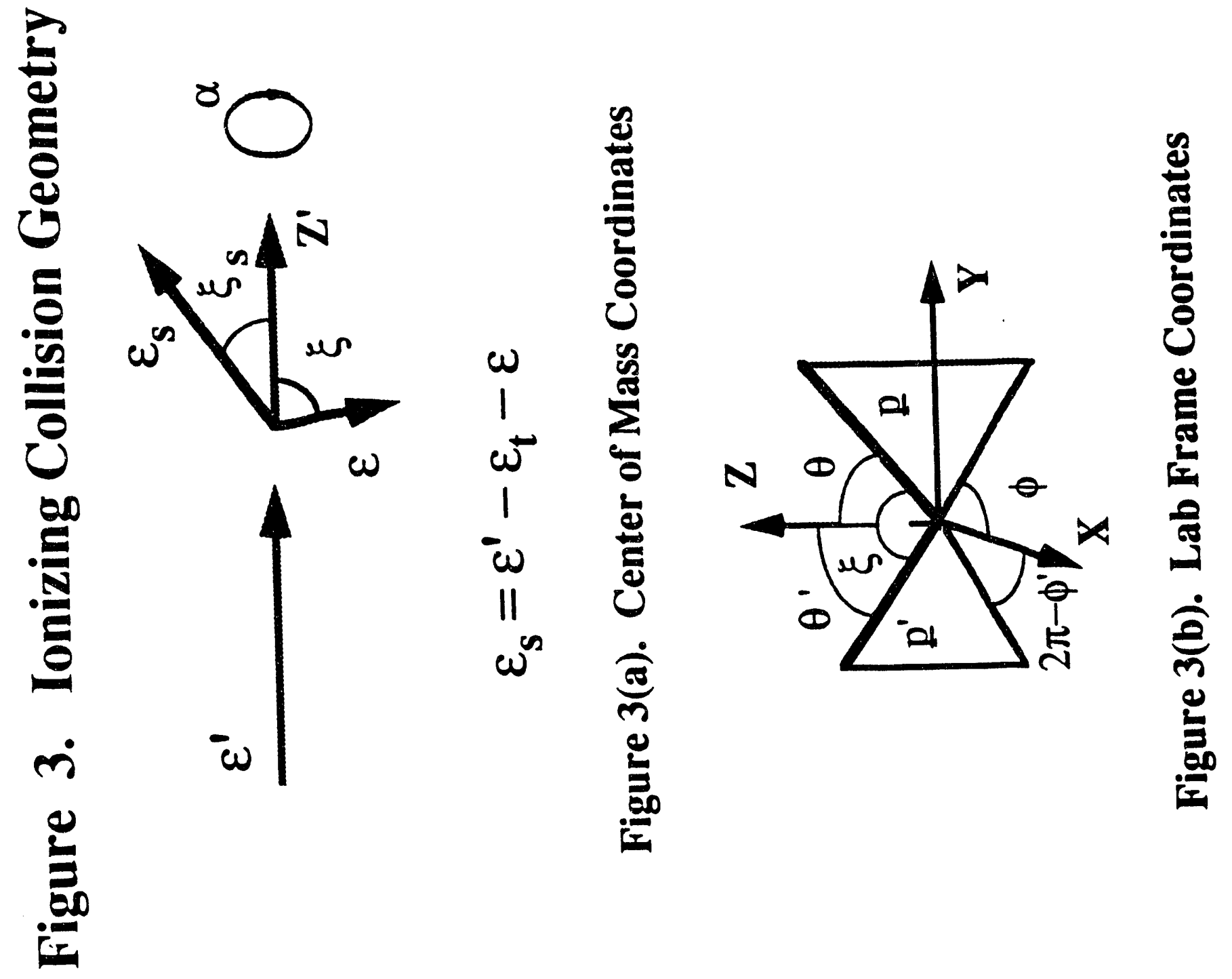


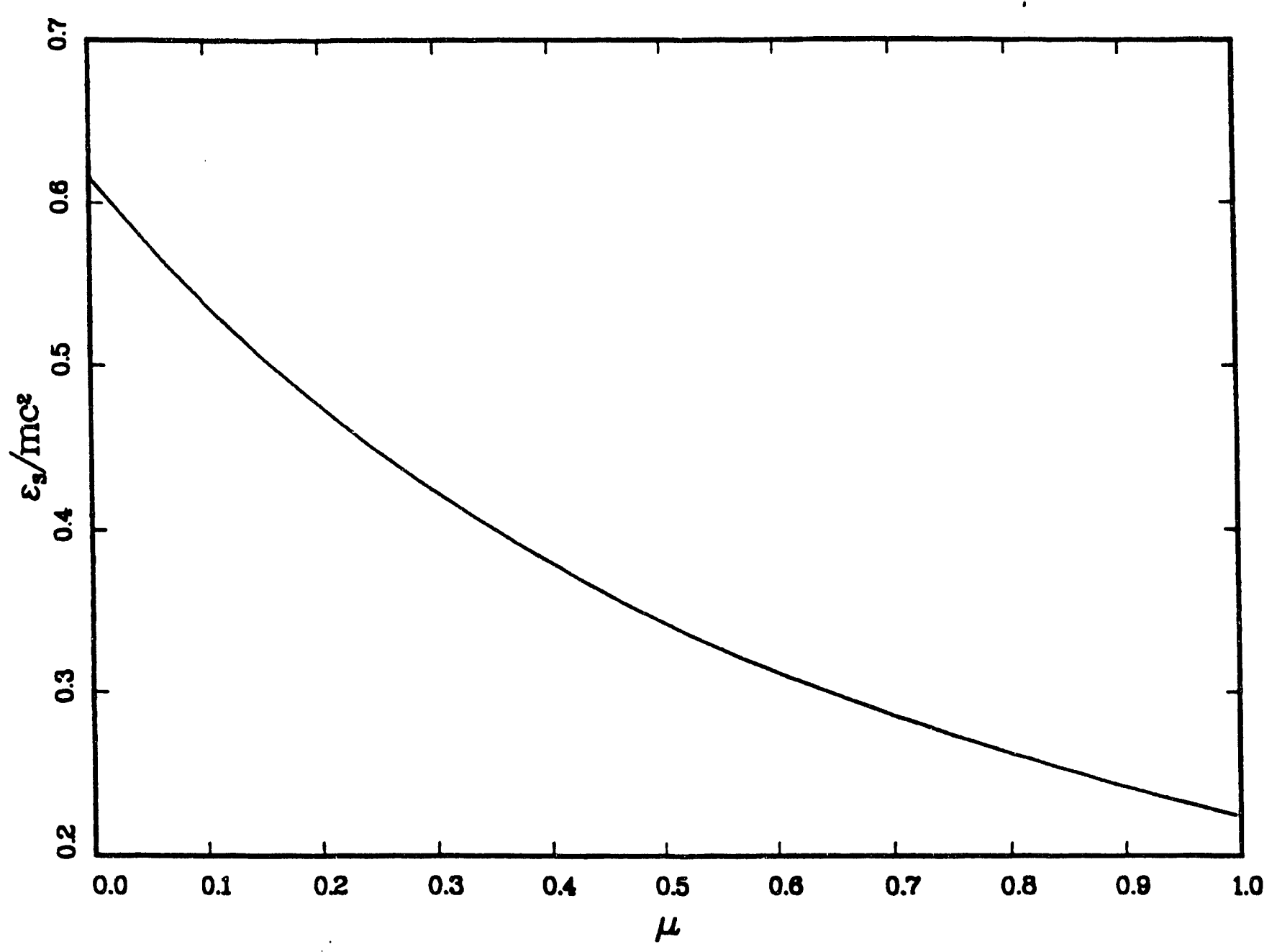

Figure 5. Relativistic Speparatrix $\delta_{0}=2$. 


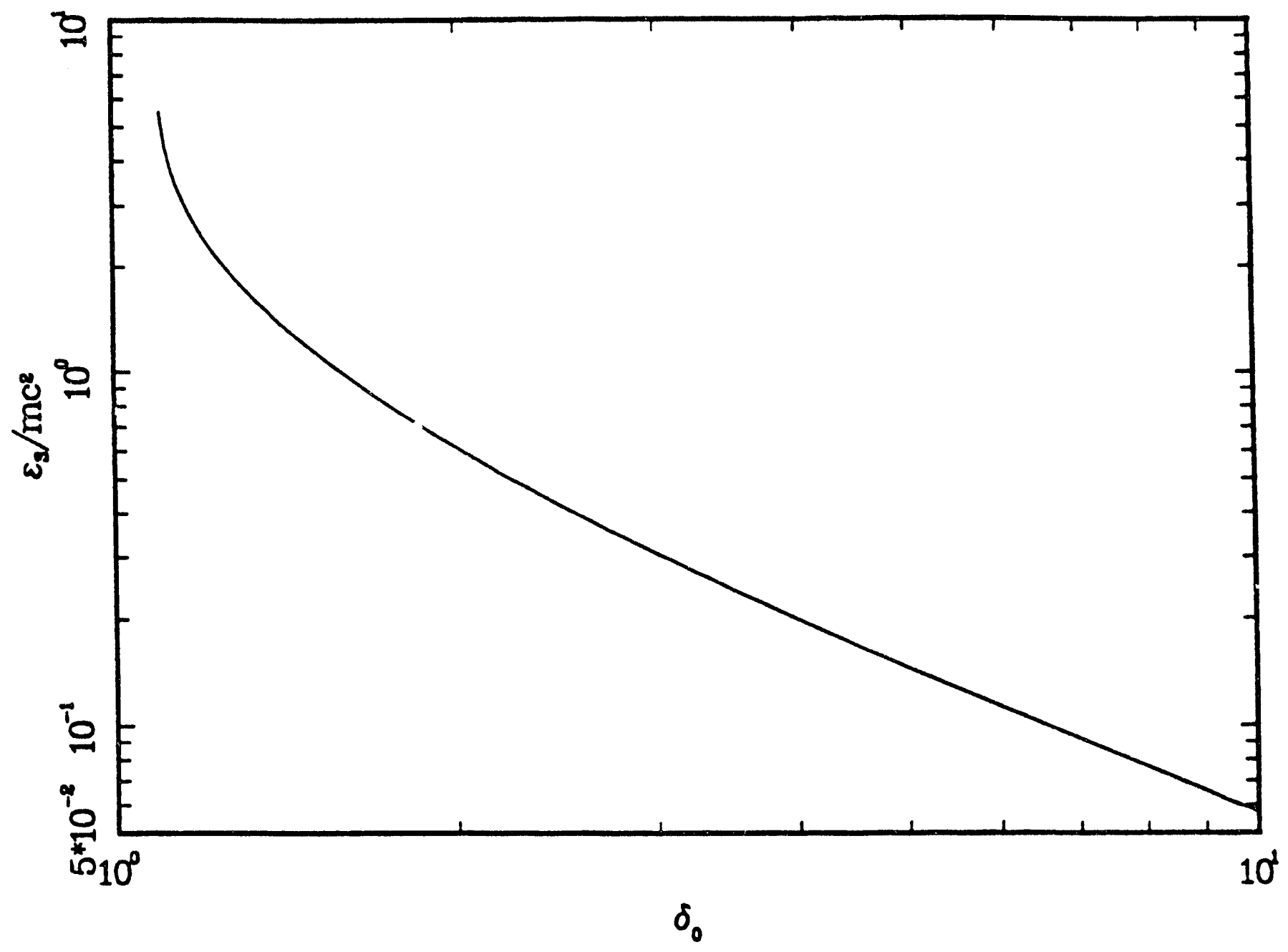

Figure 6. Minimum energy for runaway

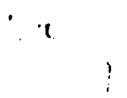




\section{Minimum Energy for Runaway SEPARATRIX}

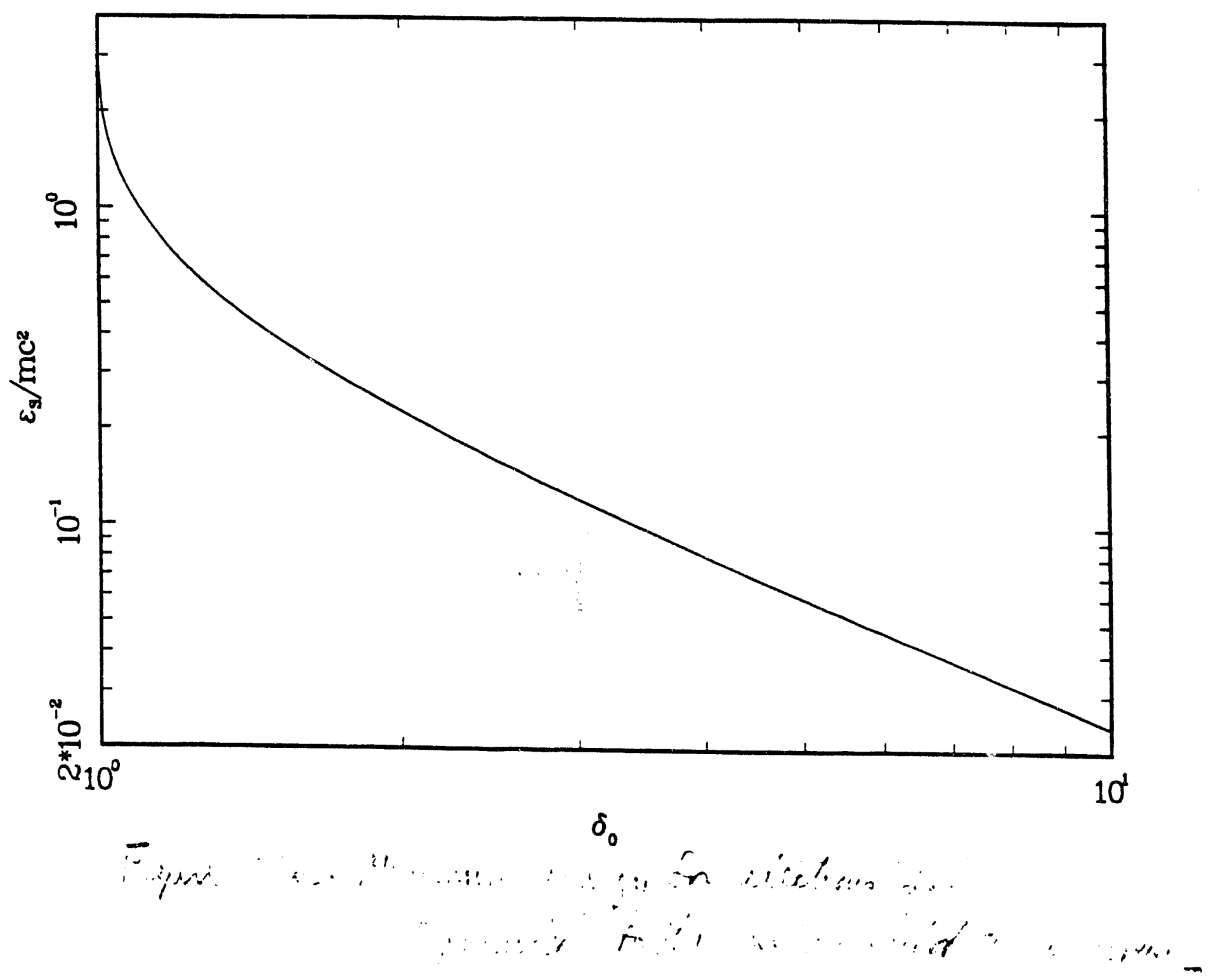




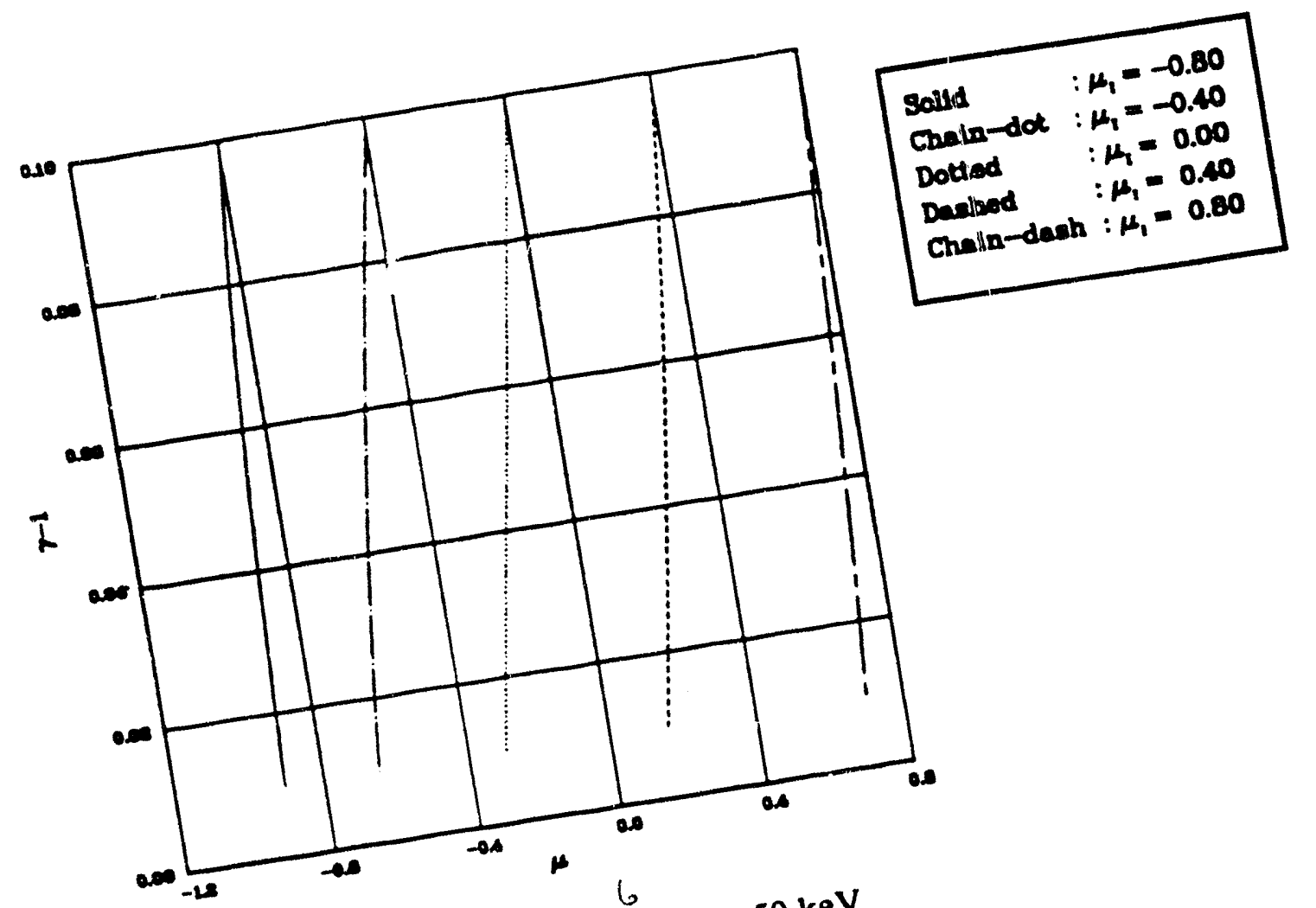

Figure 7a. $\varepsilon_{i}=50 \mathrm{keV}$.

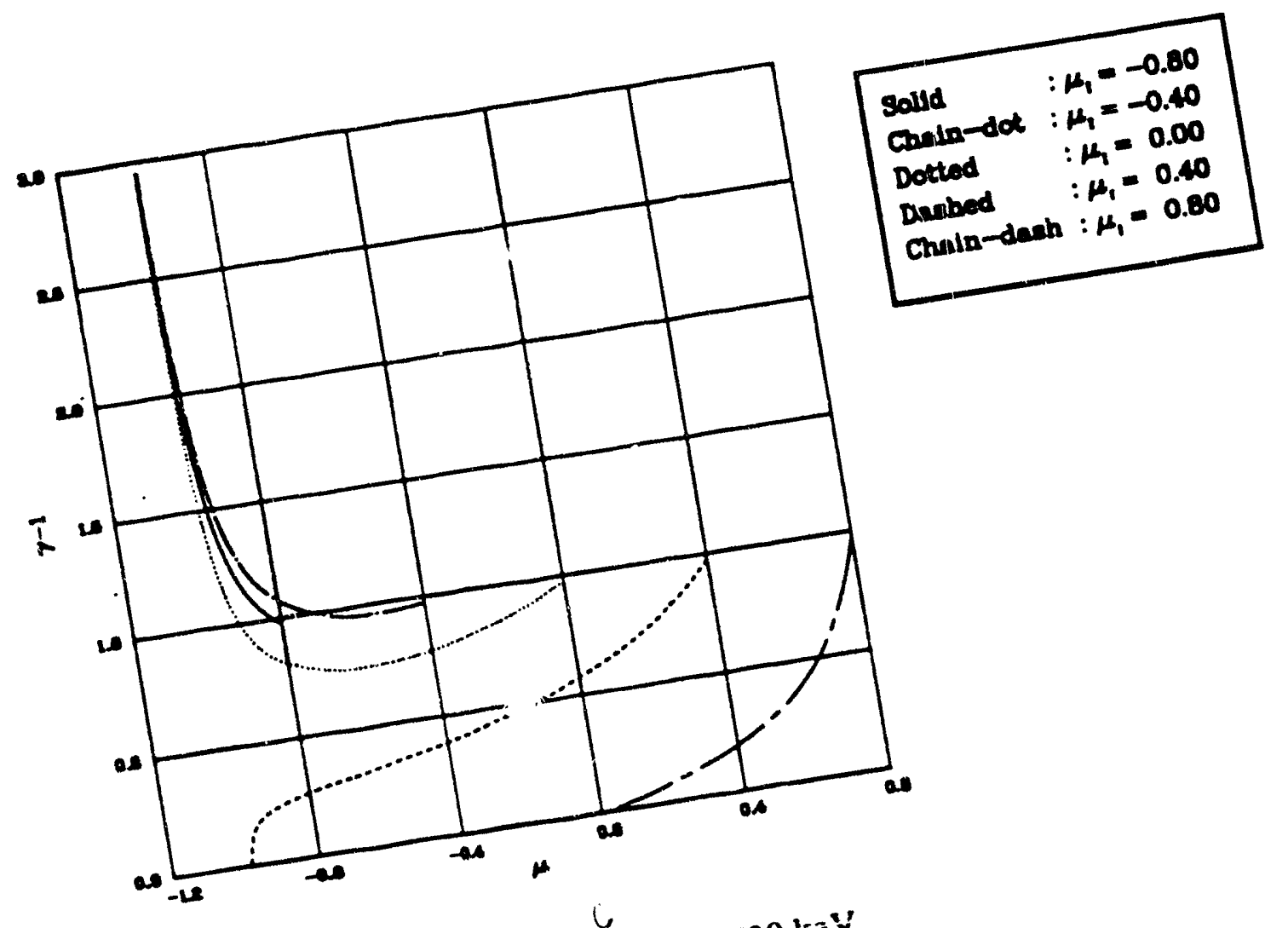

Figuie it. $\varepsilon_{i}=500 \mathrm{kev}$. 


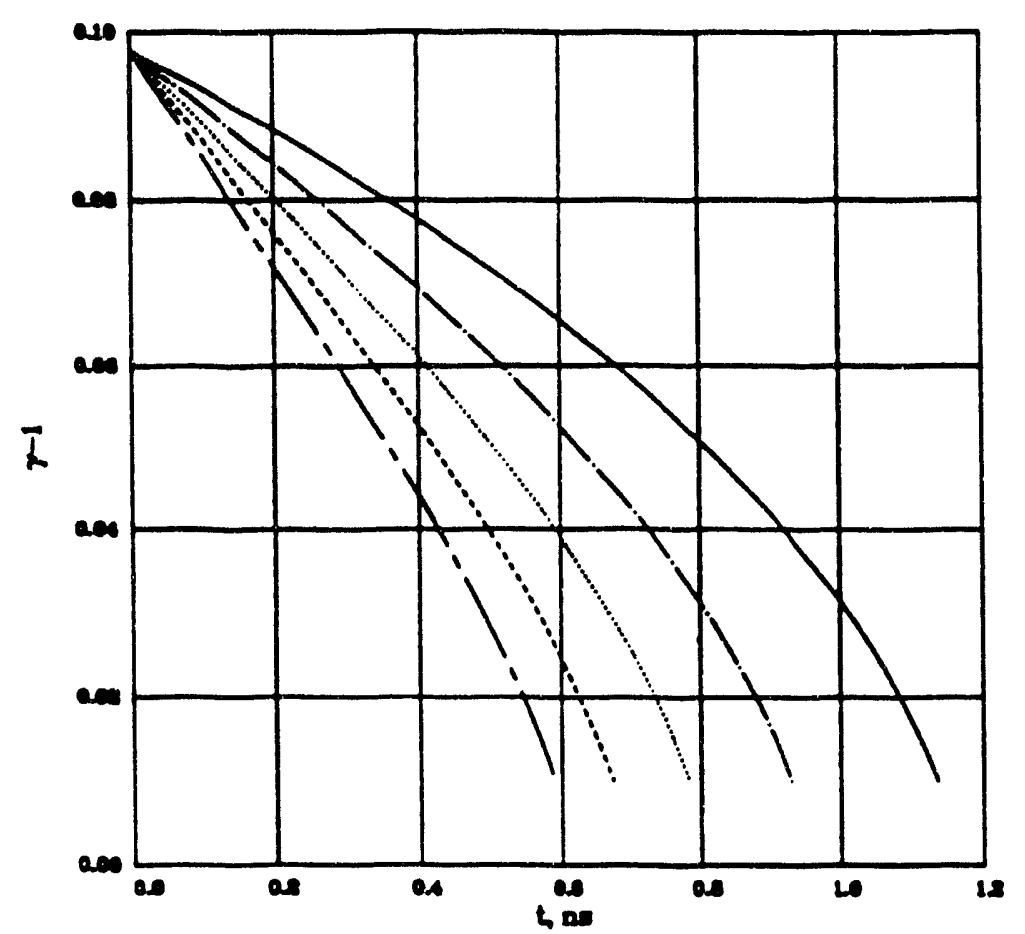

Figure \&. ?. $\varepsilon_{\mathrm{i}}=50 \mathrm{keV}$.

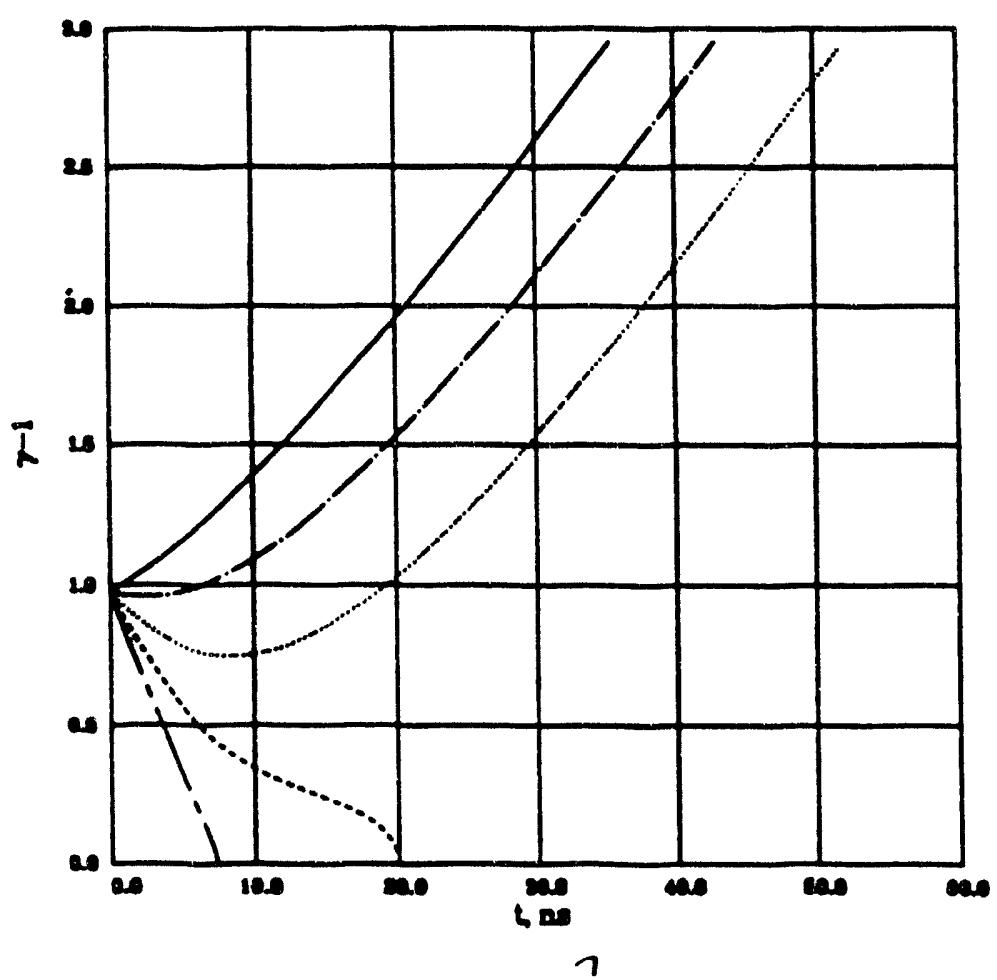

Figure $\not b \mathrm{~b} . \varepsilon_{\mathrm{i}}=500 \mathrm{keV}$
Solld $: \mu_{1}=-0.80$

Chain-dot : $\mu_{1}=-0.40$

Dotted $\quad: \mu_{1}=0.00$

Danbed $\quad: \mu_{1}=0,40$

Chaln-dach : $\mu_{1}=0.80$

$$
\begin{array}{ll}
\text { Solld } & : \mu_{1}=-0.80 \\
\text { Chaln-dot } & : \mu_{1}=-0.40 \\
\text { Dotted } & : \mu_{1}=0.00 \\
\text { Danled } & : \mu_{1}=0.40 \\
\text { Chaln-danh }: \mu_{1}=0.80
\end{array}
$$




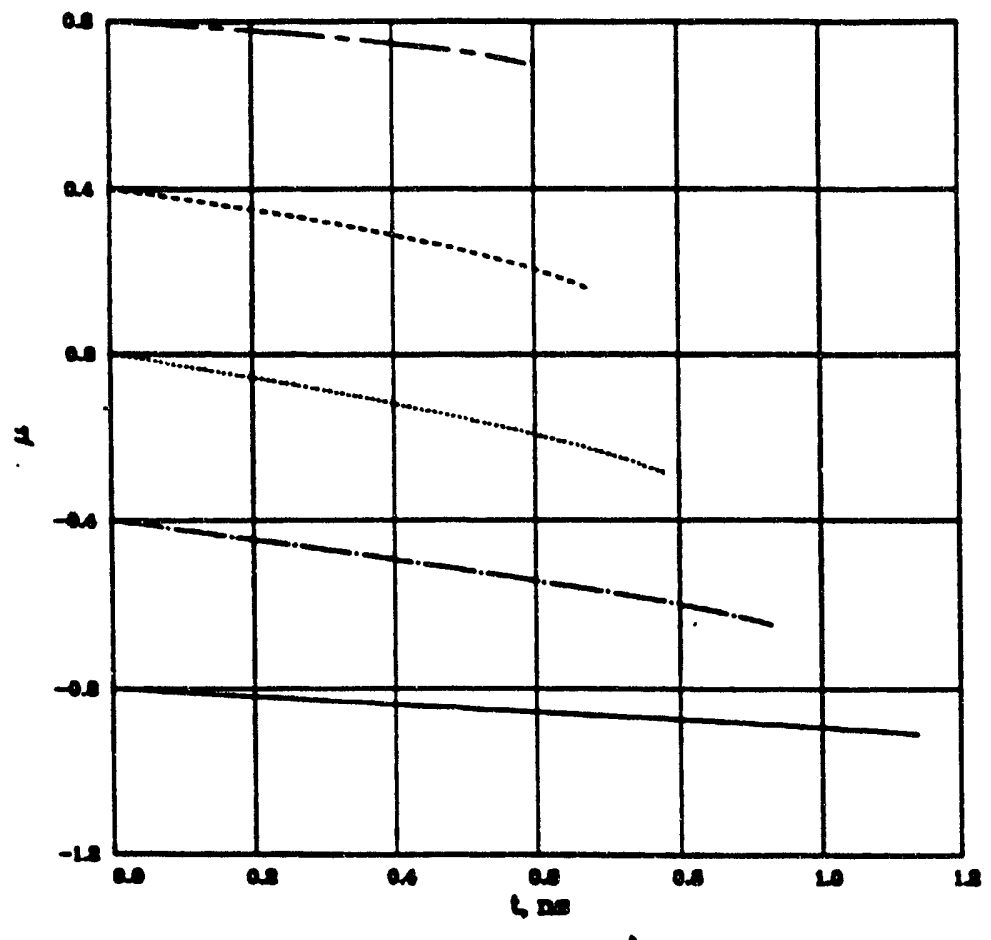

9

Figure $9 \mathrm{a} . \varepsilon_{\mathrm{i}}=50 \mathrm{keV}$.

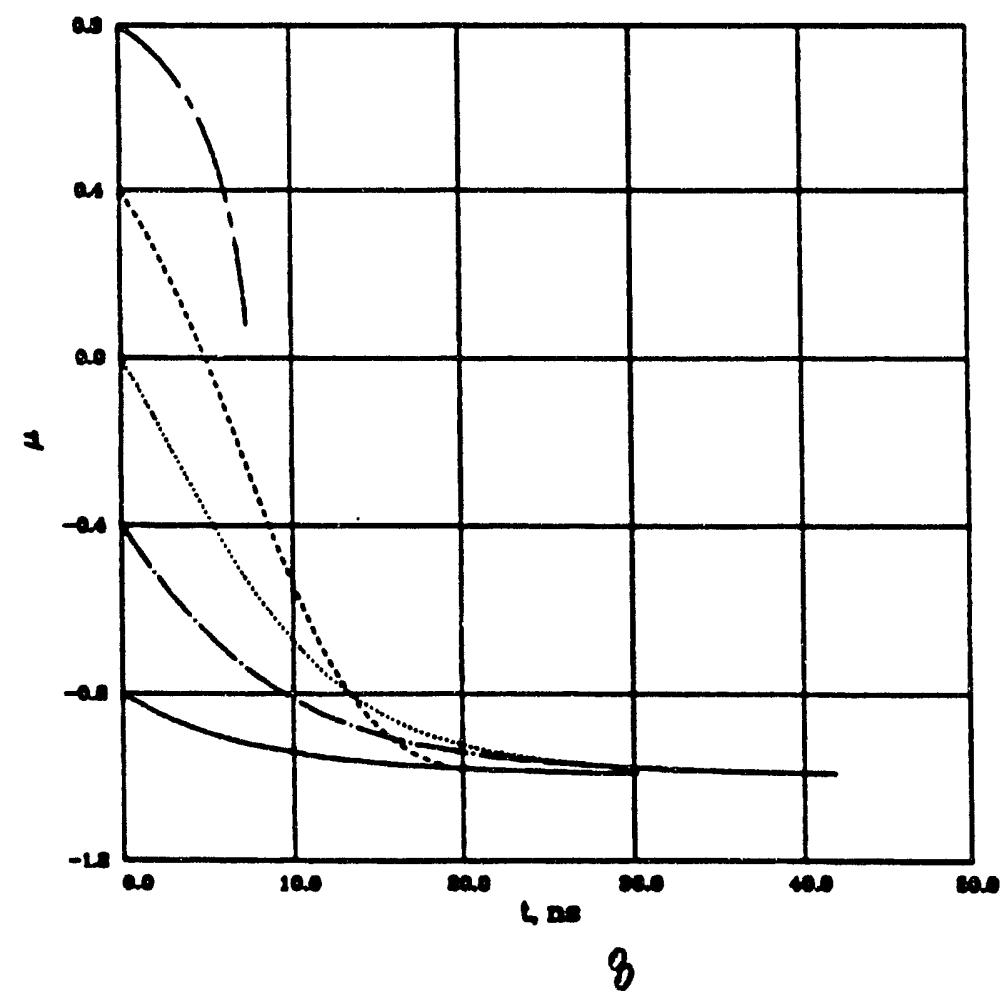

Figure \$b. $\varepsilon_{i}=500 \mathrm{keV}$.
Solld $\quad: \mu_{1}=-0.80$

Chaln-dot : $\mu_{1}=-0.40$

Dotted $\quad: \mu_{1}=0.00$

Danbed : $\mu_{1}=0.40$

Chaln-dash $: \mu_{1}=0.80$ $\begin{array}{ll}\text { Solld } & : \mu_{1}=-0.80 \\ \text { Chaln-dot } & : \mu_{1}=-0.40 \\ \text { Dotted } & : \mu_{1}=0.00 \\ \text { Danbed } & : \mu_{1}=0.40 \\ \text { Chaln-dash }: \mu_{1}=0.80\end{array}$ 


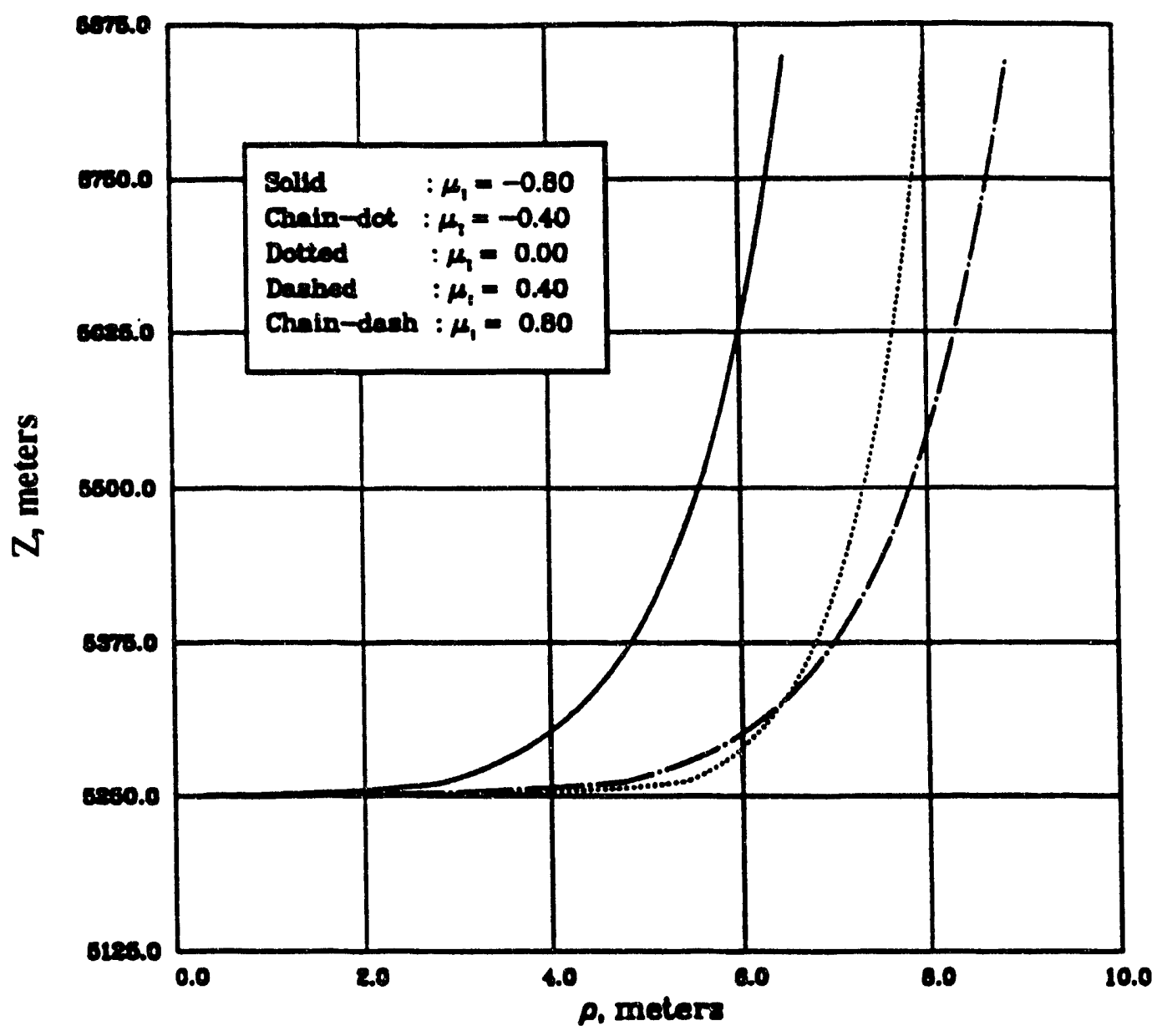

a

Figure 10. Altitude vs range tracjectory $\varepsilon_{\mathrm{i}}=500 \mathrm{keV}$ and $\delta_{0}=2$. 


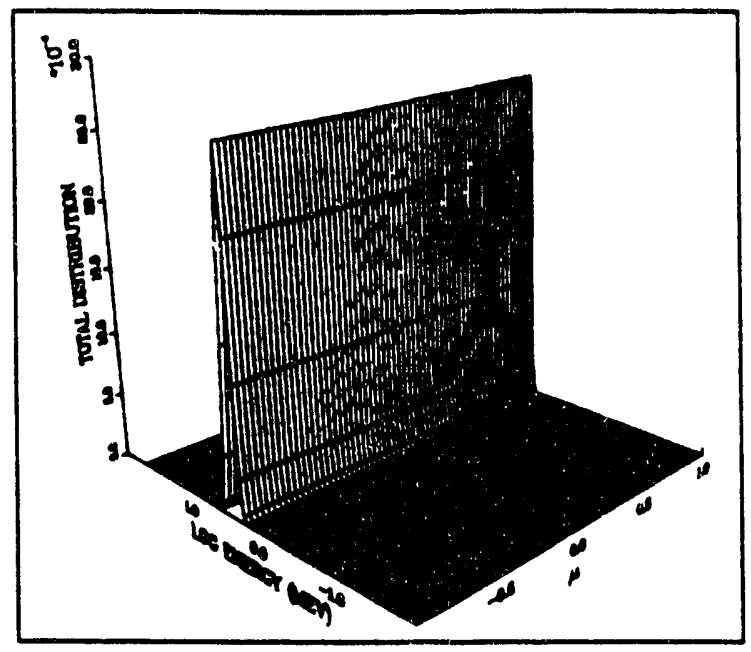

Figure 1 1a. $\mathrm{t}=0.00 \mathrm{~ns}$ 10

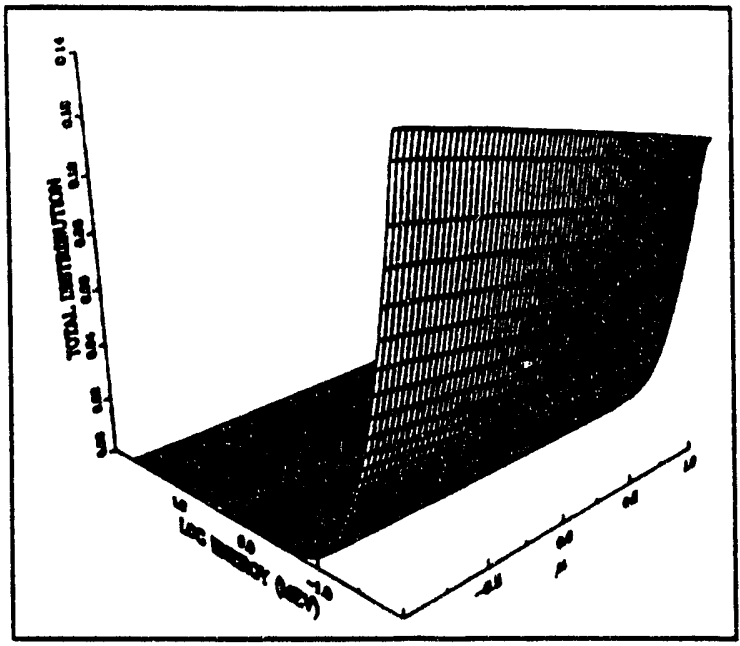

Figure $k \mathrm{~b} . t=1.00 \mathrm{~ns}$ 10

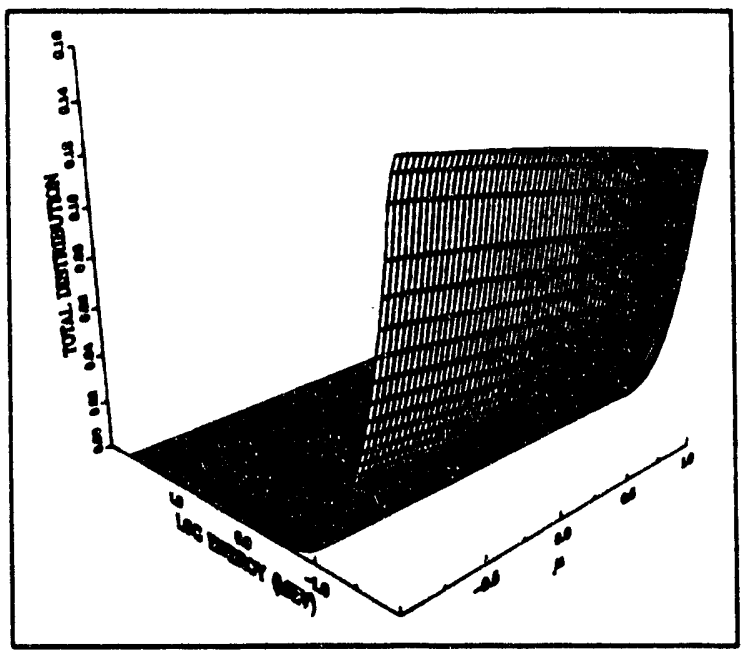

Figure $\backslash 1 \mathrm{c} . t=1.50 \mathrm{~ns}$ 10

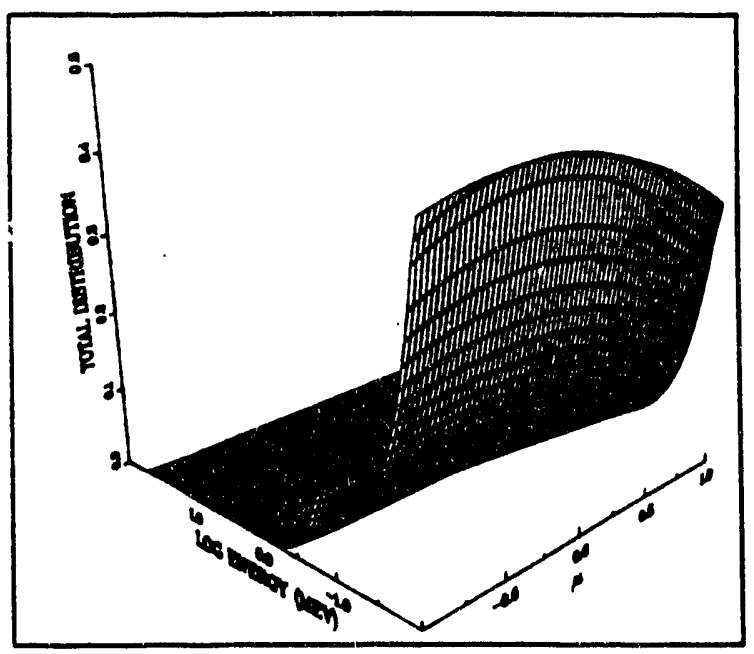

Figure Kd. $\mathrm{t}=20.0 \mathrm{~ns}$ 10

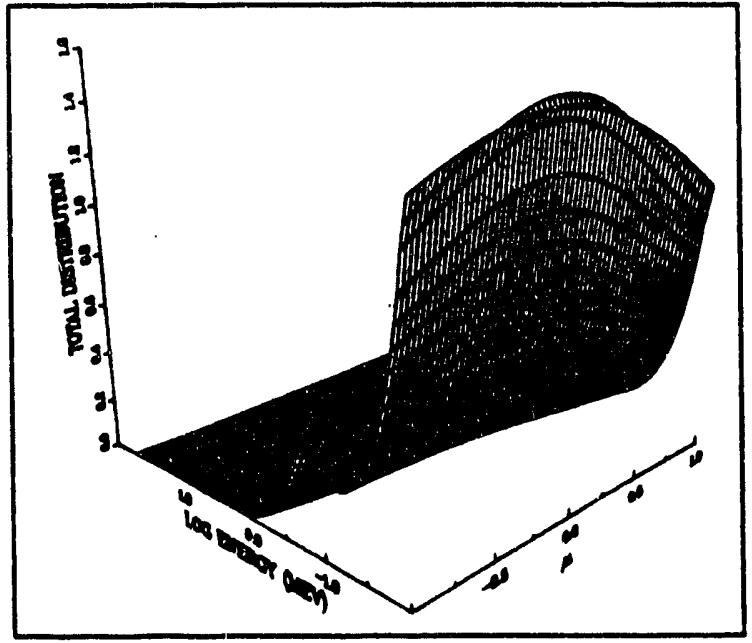

Figure Ȟe. $t=50.0 \mathrm{~ns}$ 10

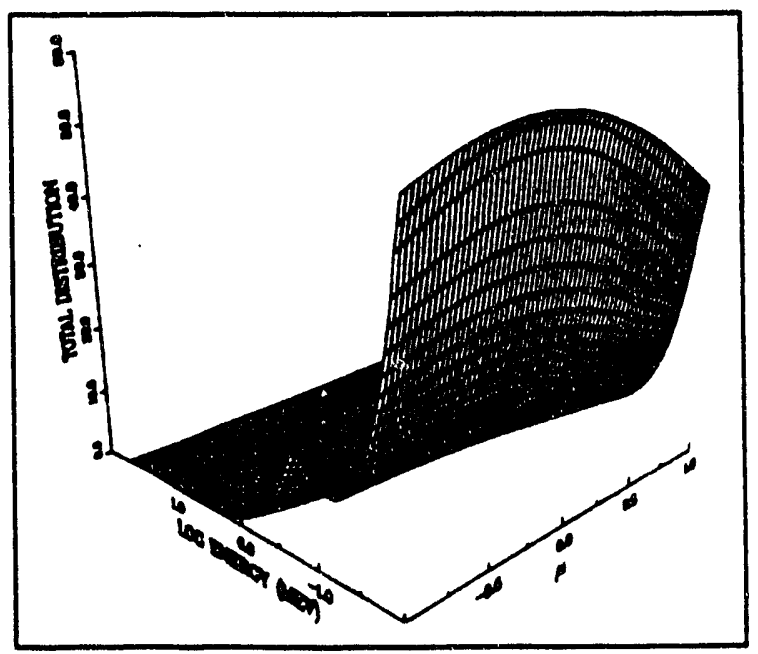

Figure $\not 1$ f. $t=150.0 \mathrm{~ns}$ 10 


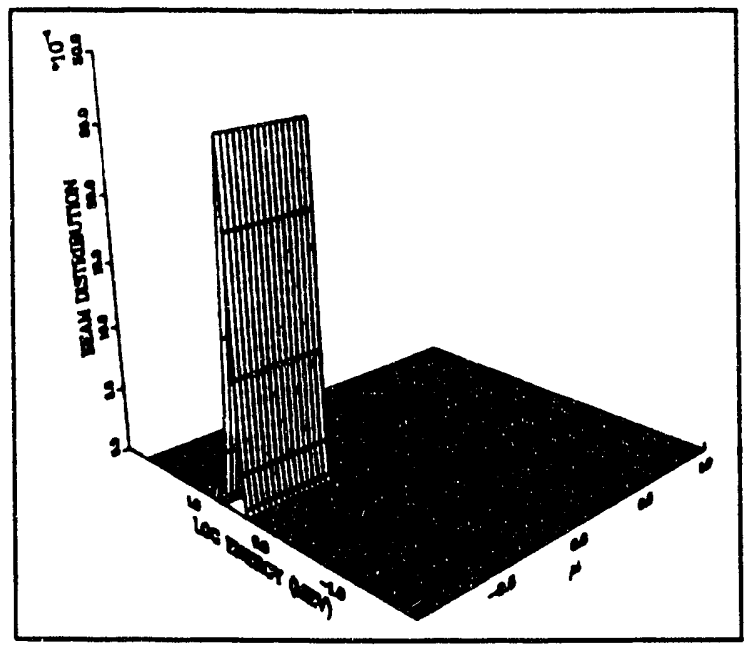

Figure 12a. $t=0.00$ ns.

11

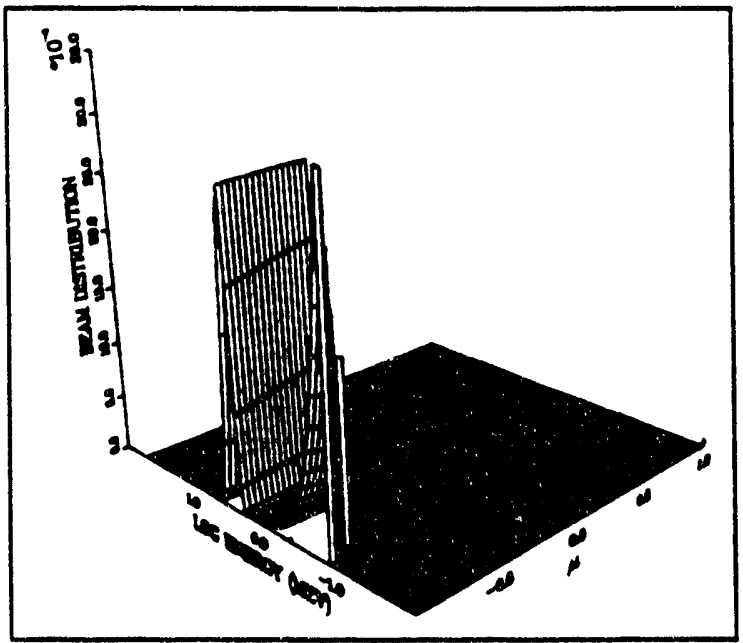

Figure 126. $\mathrm{t}=1.00$ ns. (1)

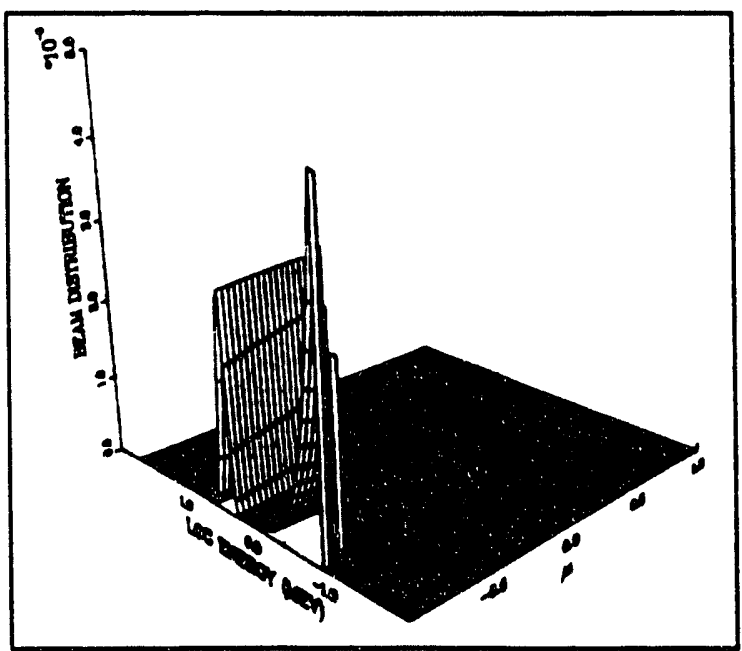

Figure 12 c. $t=1.50$ ns.

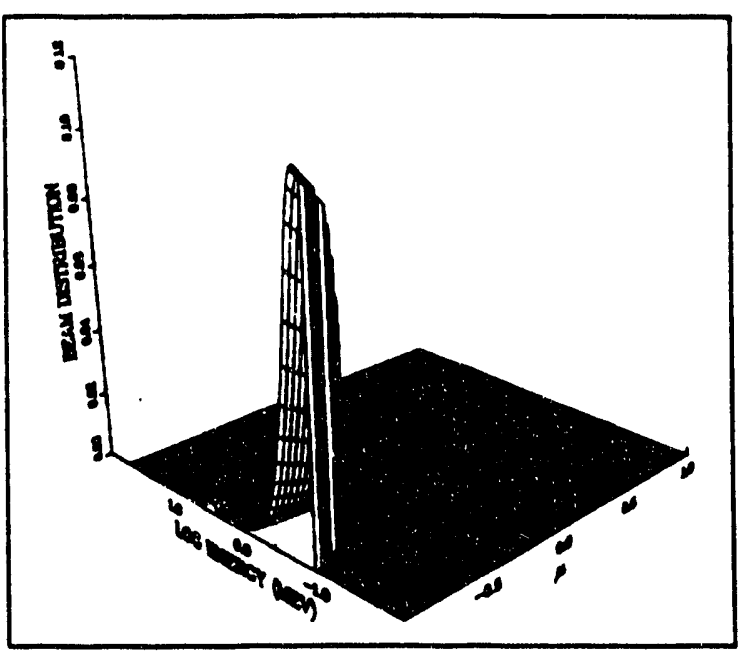

Figure 12d. $t=20.0$ ns. (1)

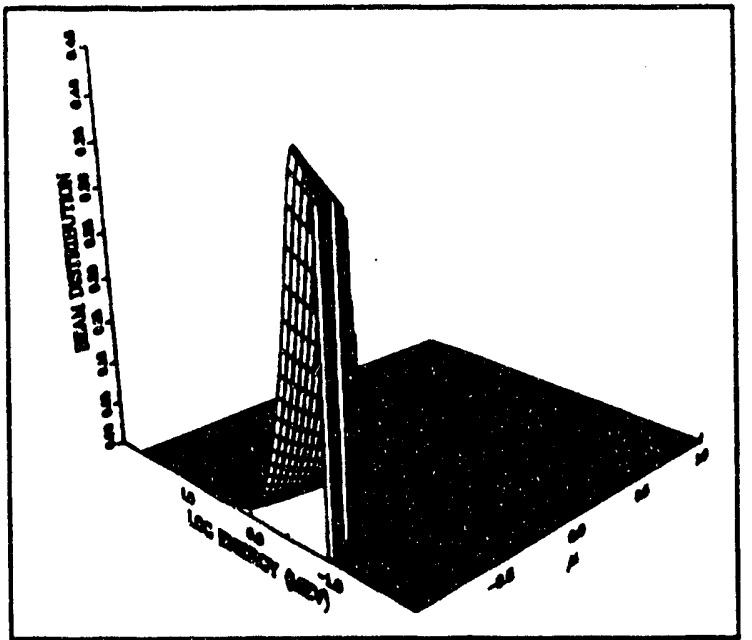

Figure 12e. $t=50.0$ ns.

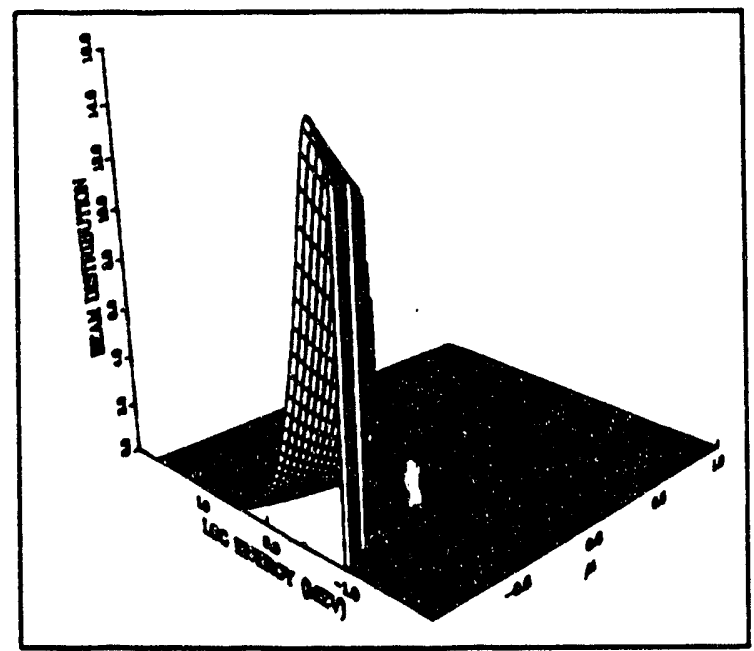

Figure Kf. $t=150.0 \mathrm{~ns}$. 


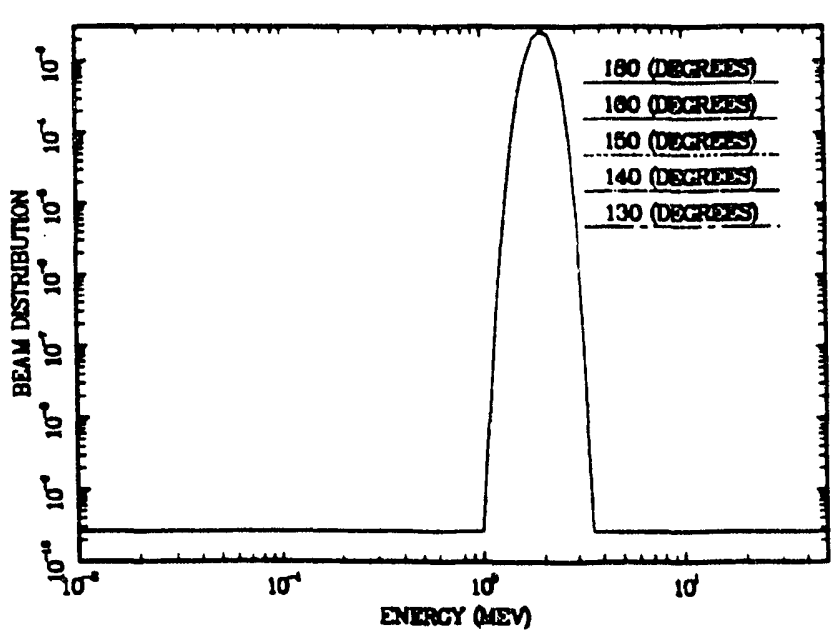

Figure 13a. $\mathrm{t}=0.00 \mathrm{~ns}$.

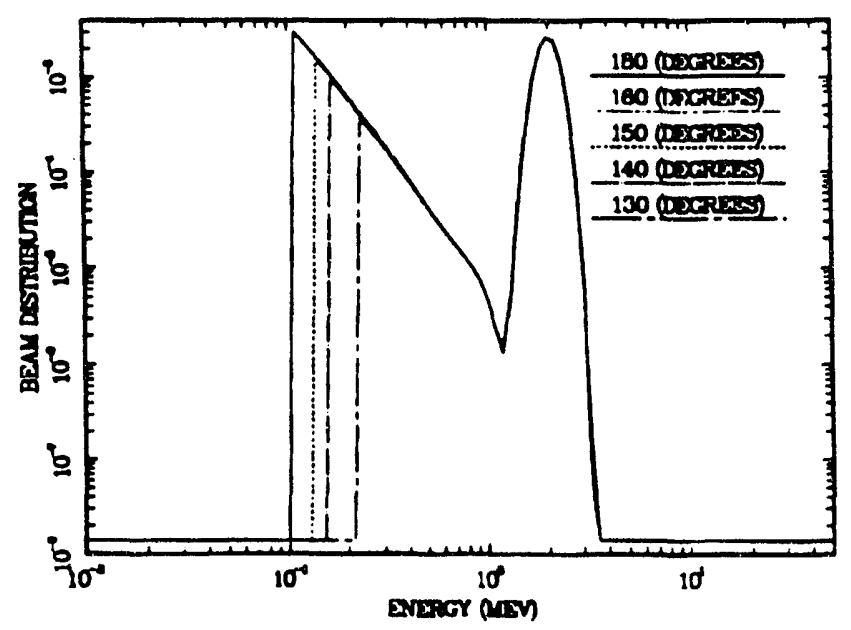

Figure bbo. $t=1.00$ ns.

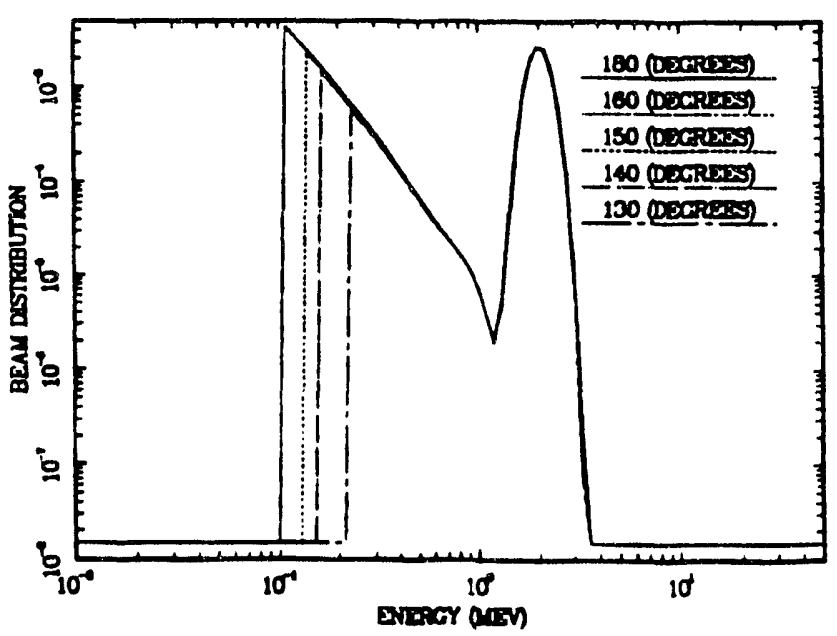

Figure $1 x^{\prime} c . t=1.50 \mathrm{~ns}$.

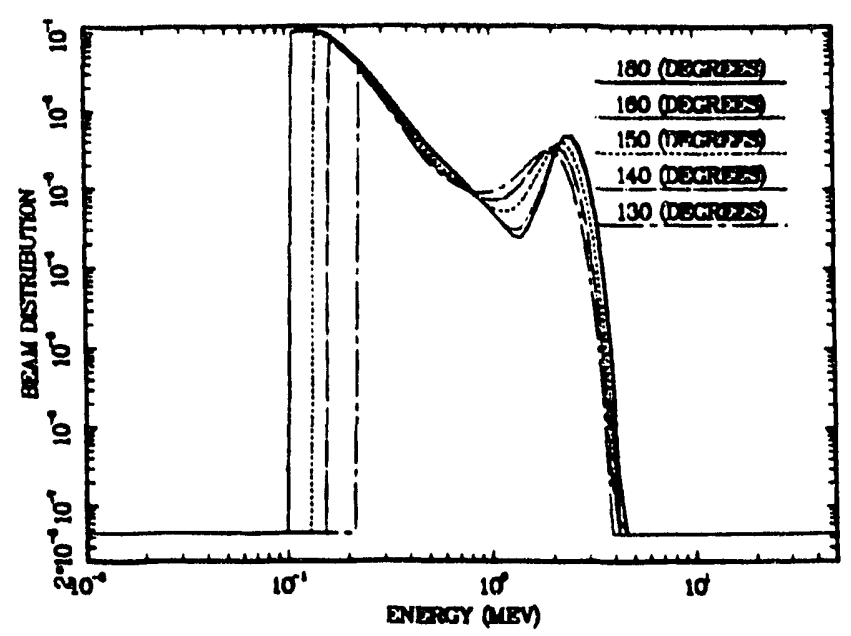

Figure 13d. $t=20.0 \mathrm{~ns}$.

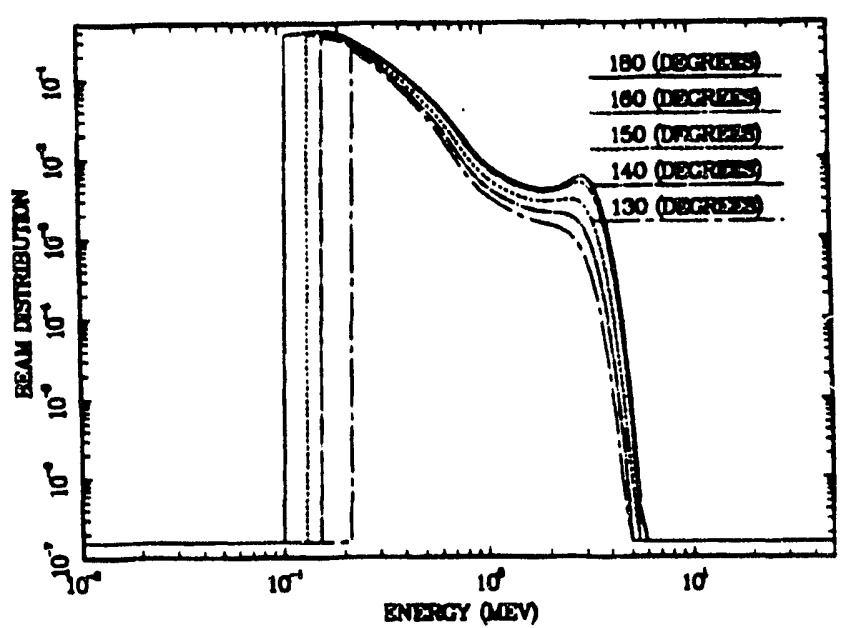

Figure 1ß̌e. $t=50.0$ ns.

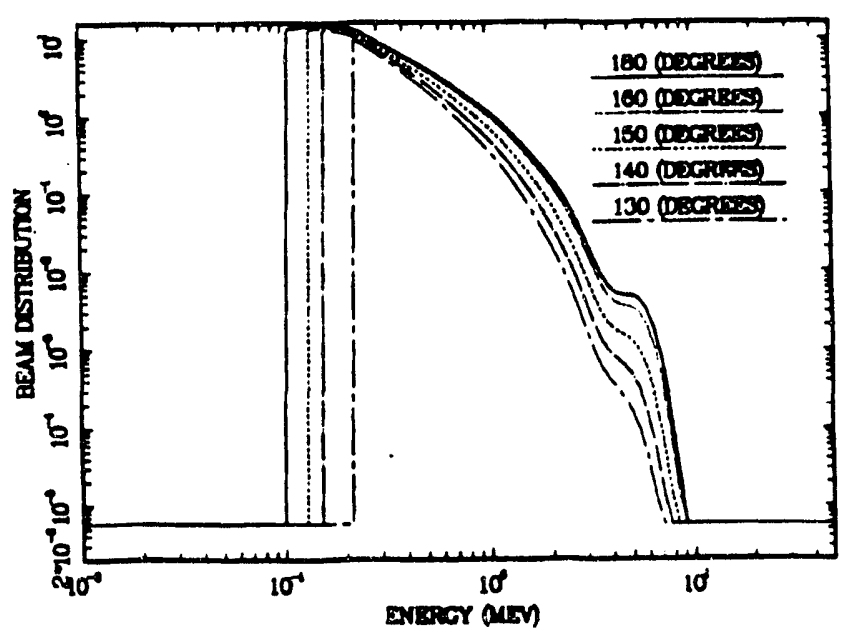

Figure 13f. $t=150.0 \mathrm{~ns}$. 


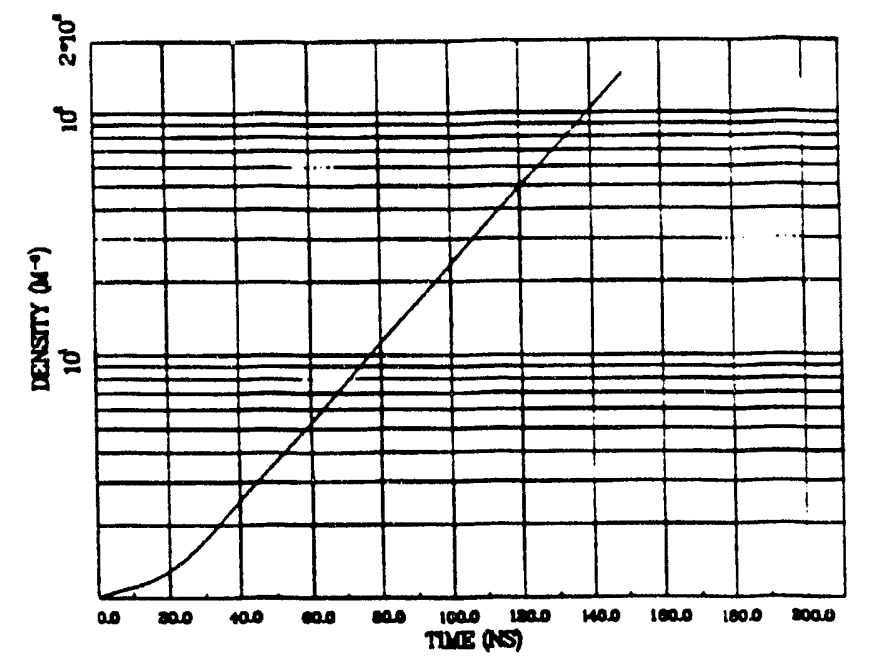

Figure 14a. Electron density. 13

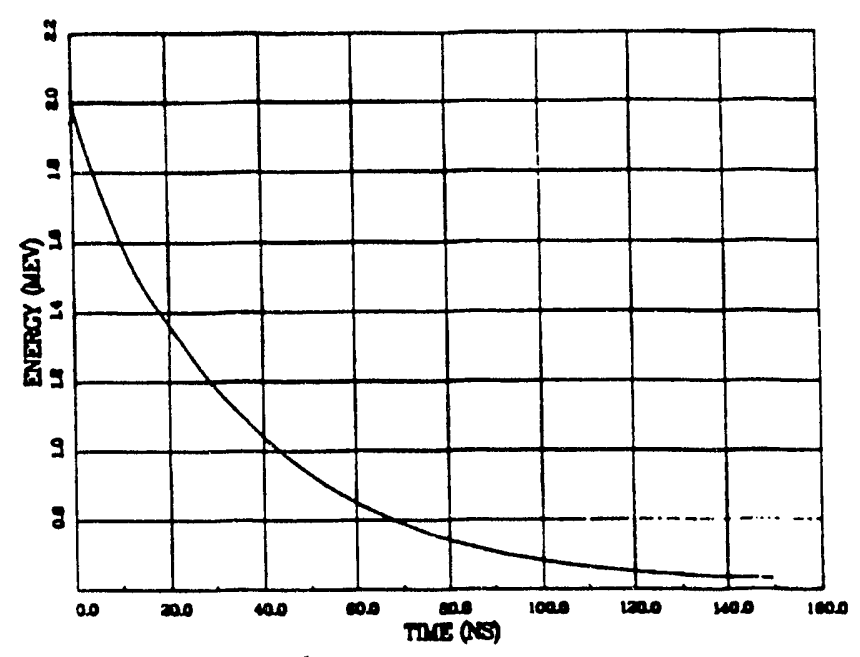

Figure $14 \mathrm{~b}$. Average kinetic energy. 13

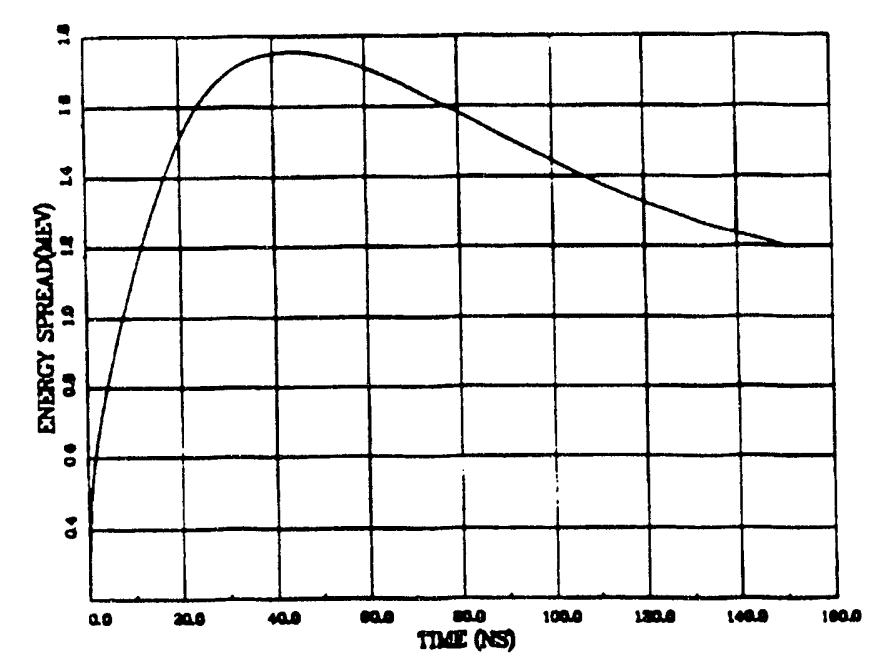

Figure 14c. Kinetic energy spread. i?

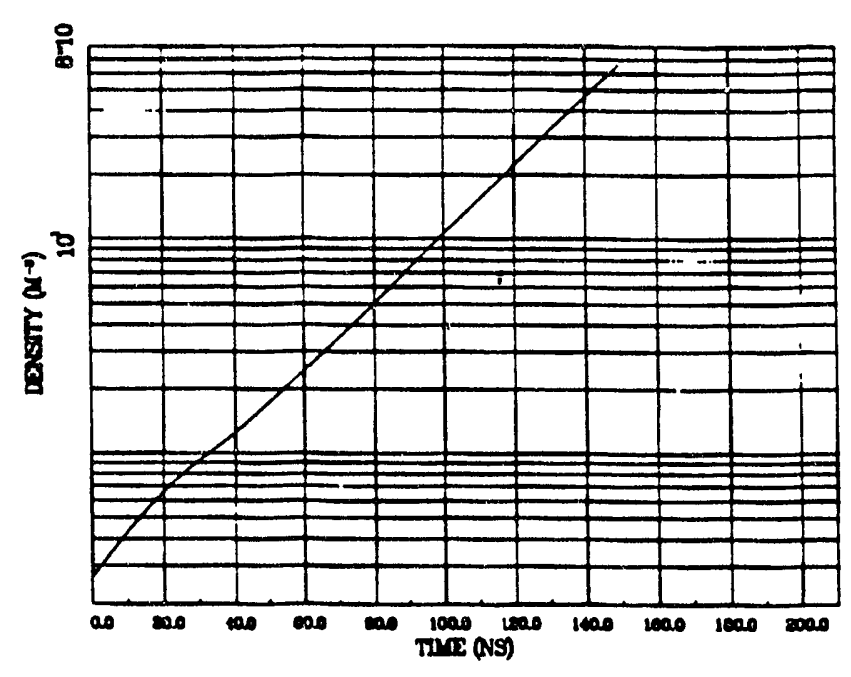

Figure 14d. Beam density. 13

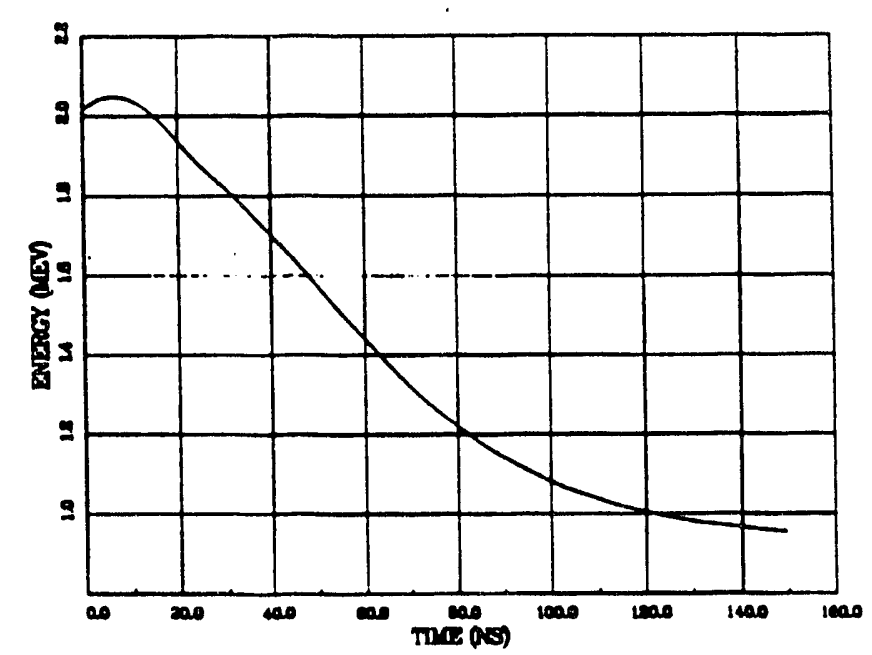

Figure 14e. Beam average kinetic energy. 13

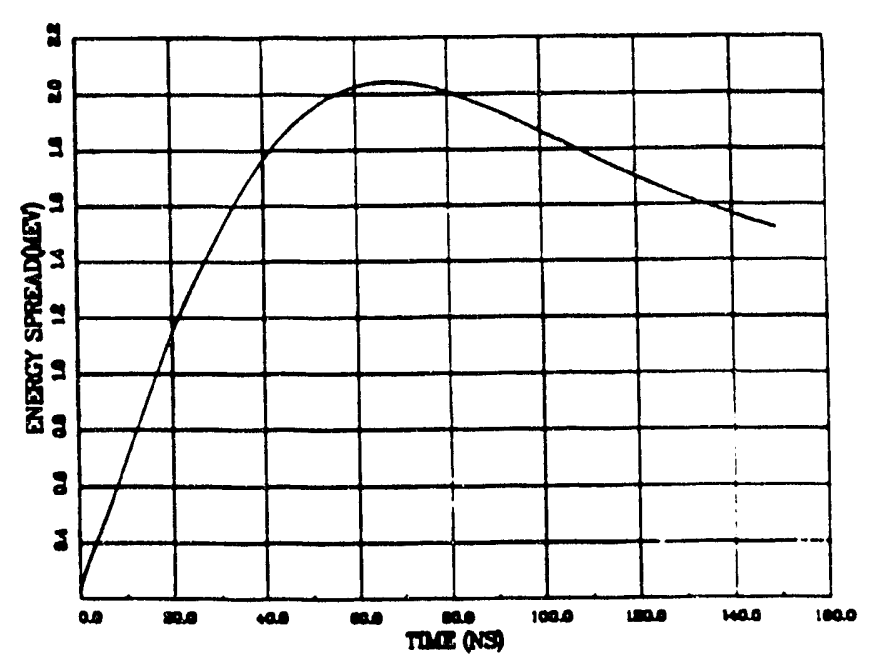

Figure 14f. Beam kinetic energy spread. 


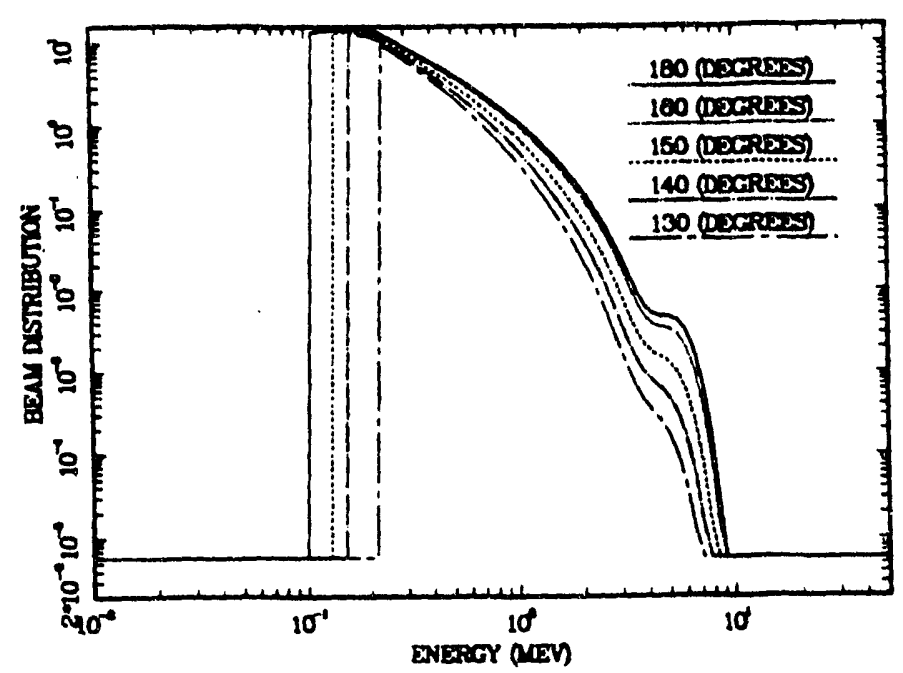

Figure $\underset{14}{16 \mathrm{a}} . \delta_{0}=2.0$.

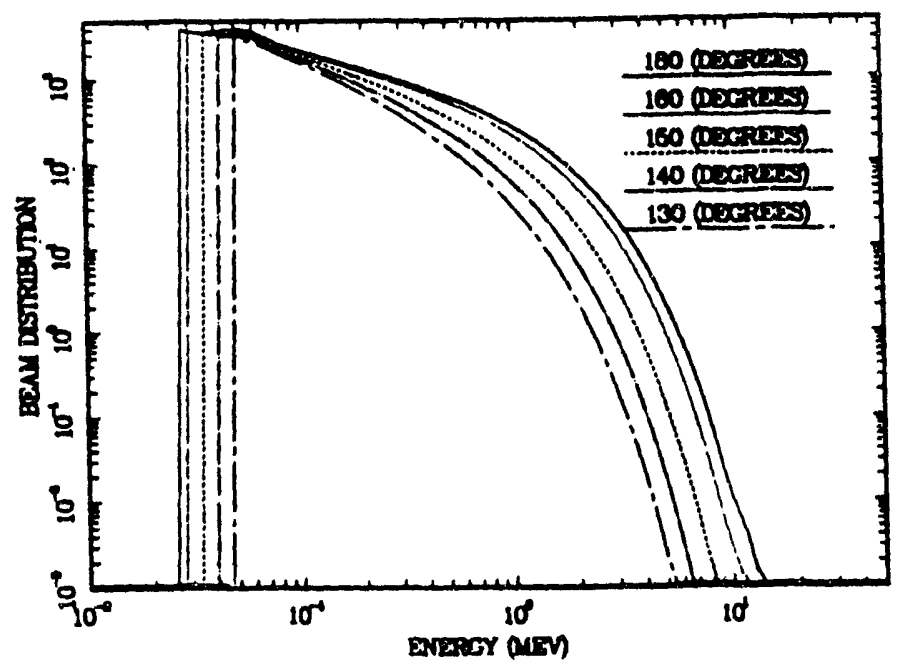

Figure $15 b . \delta_{0}=5.0$.

U

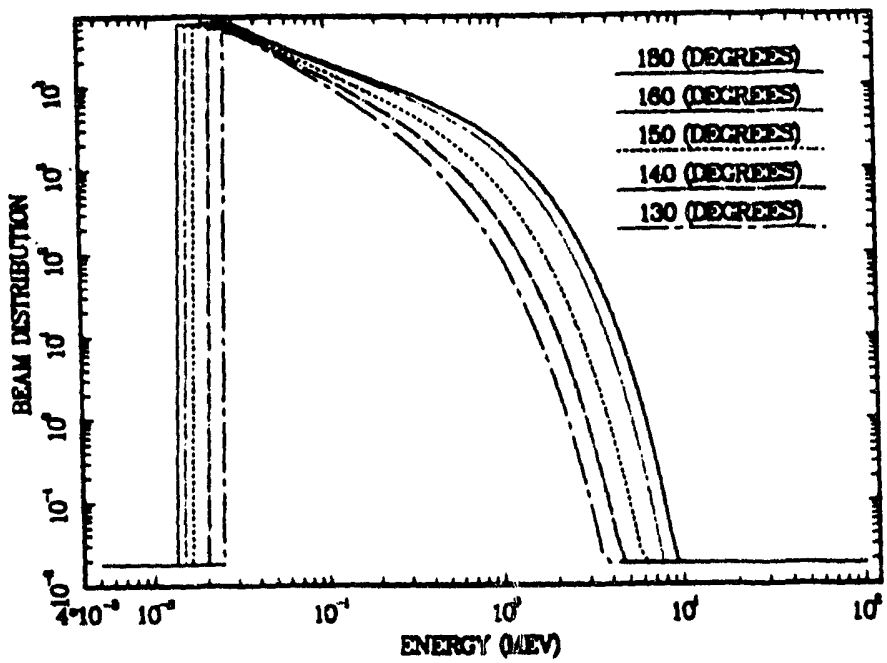

Figure $15 \mathrm{c}$. $\delta_{0}=8.0$. 


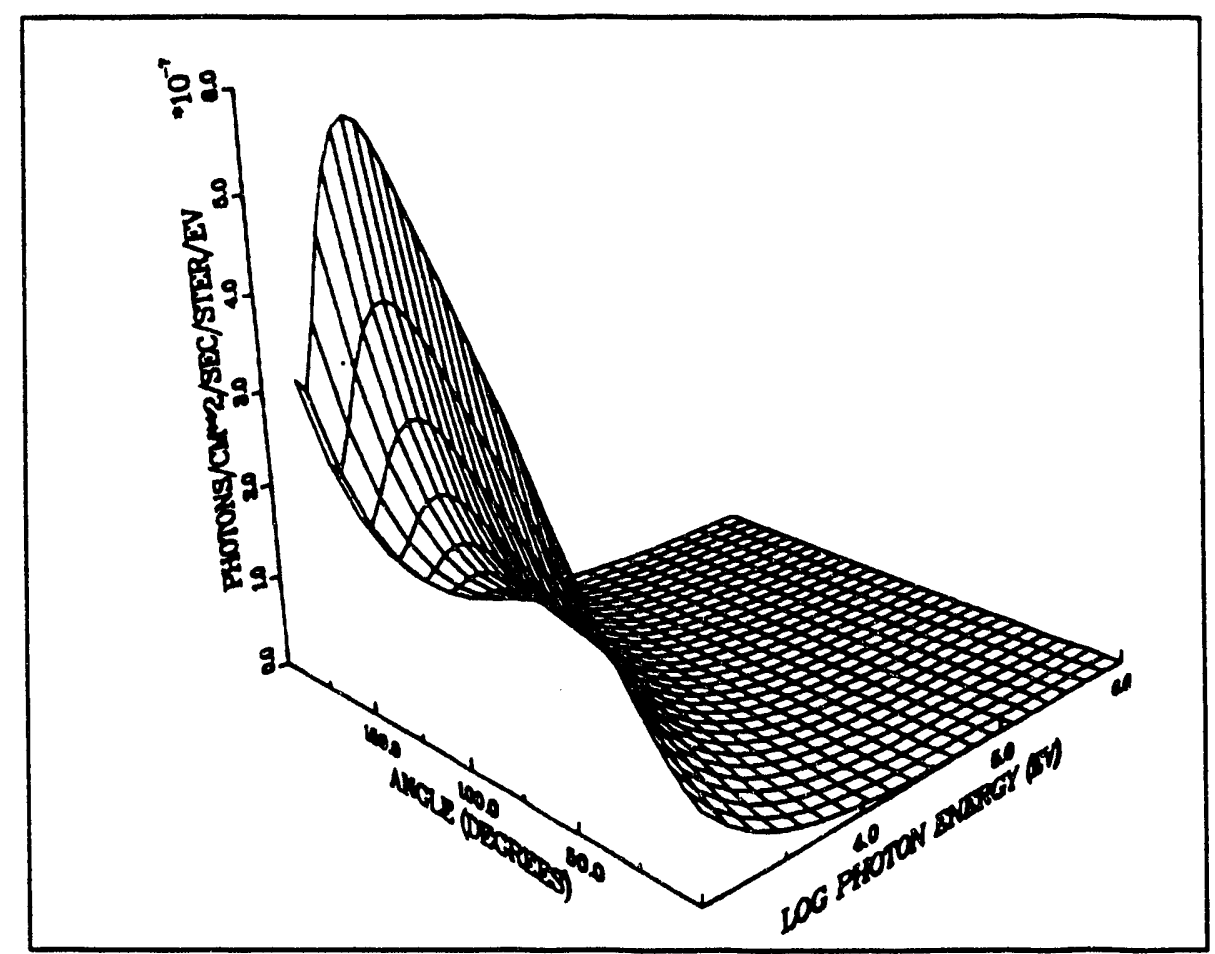

Figure 19a. Three-dimensional plot. is

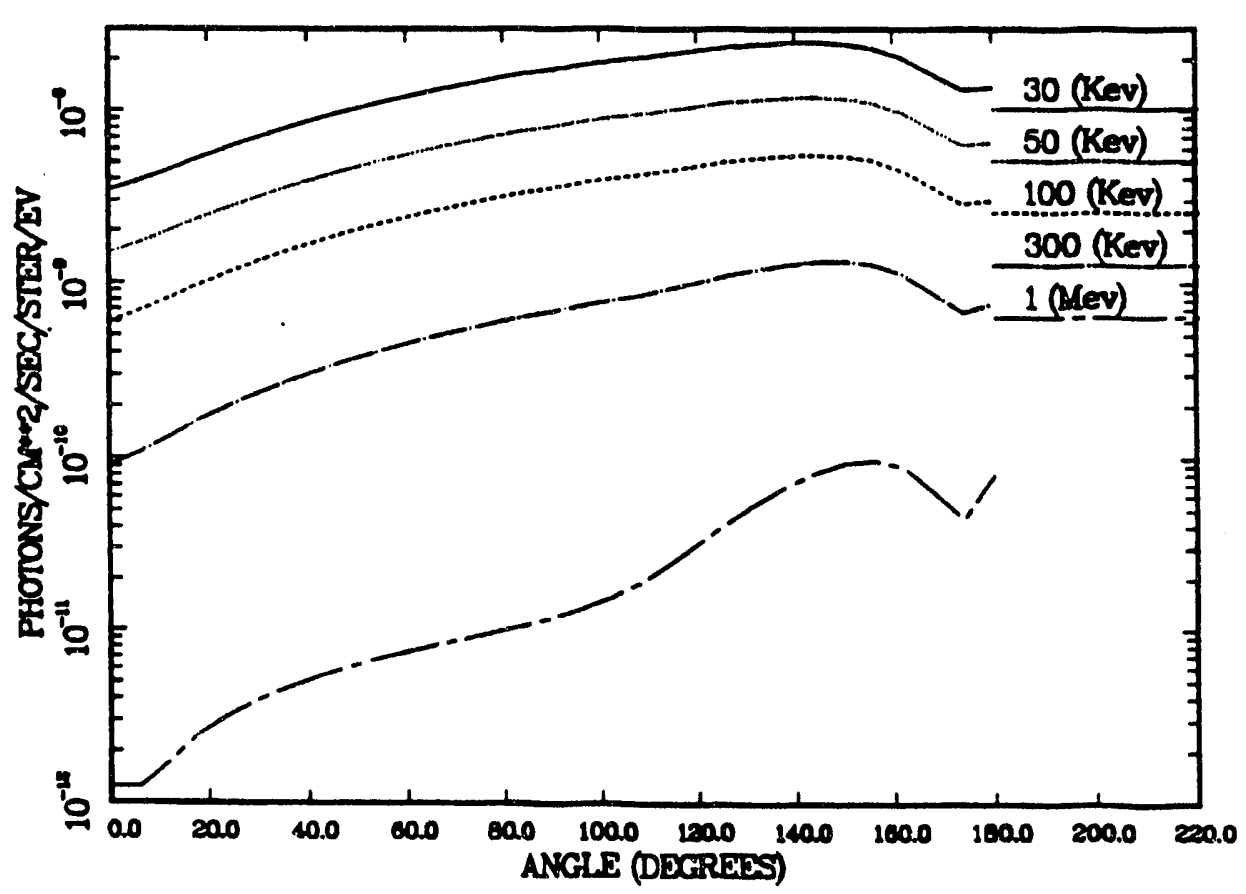

Figure $19 \mathrm{~b}$. Two-dimensional plot for various primary energies. is 

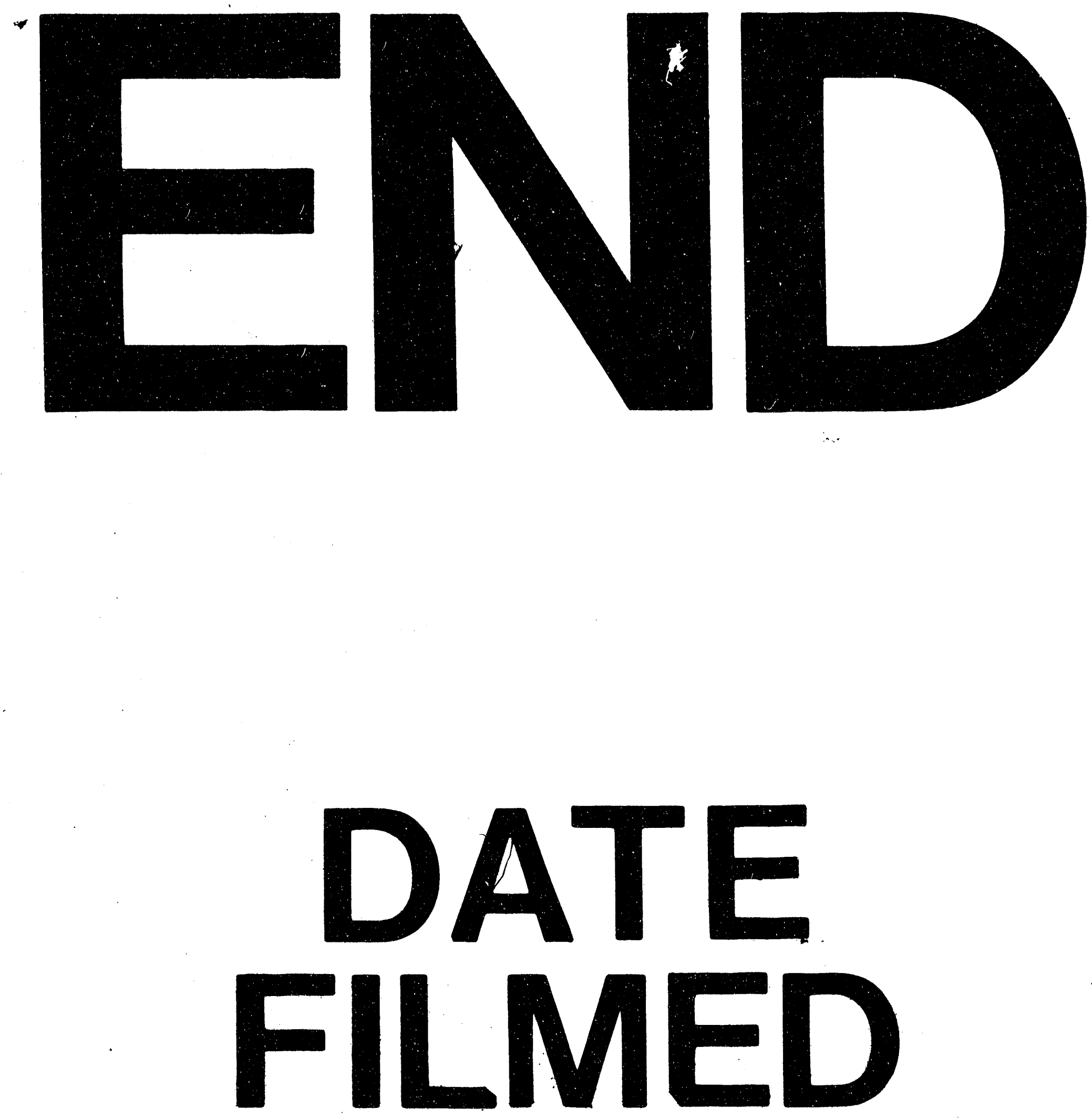

1

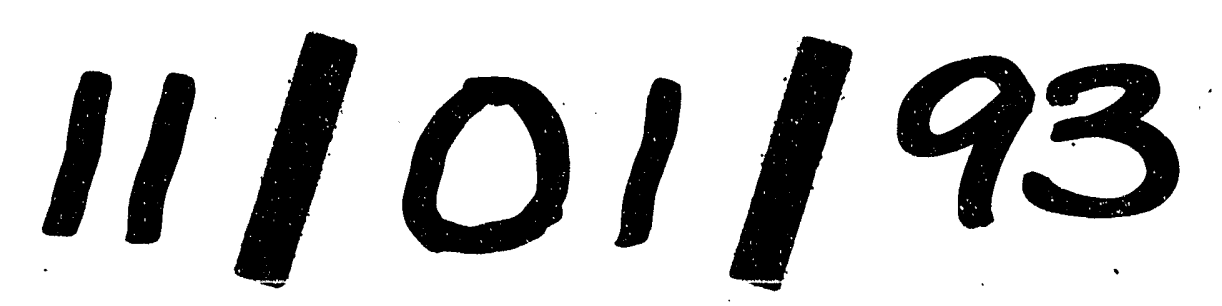


